\title{
ARITHMETICITY OF HYPERBOLIC 3-MANIFOLDS CONTAINING INFINITELY MANY TOTALLY GEODESIC SURFACES
}

\author{
G. MARGULIS AND A. MOHAMMADI
}

\begin{abstract}
We prove that if a closed hyperbolic 3-manifold $M$ contains infinitely many totally geodesic surfaces, then $M$ is arithmetic.
\end{abstract}

\section{INTRODUCTION}

Let $\mathbf{G}$ be a connected semisimple $\mathbb{R}$-group so that $\mathbf{G}(\mathbb{R})$ has no compact factors. An irreducible lattice $\Gamma_{0}$ in $\mathbf{G}(\mathbb{R})$ is called arithmetic if there exists a connected, non-commutative, almost $\mathbb{Q}$-simple, $\mathbb{Q}$-group $\mathbf{F}$ and an $\mathbb{R}$-epimorphism $\varrho: \mathbf{F} \rightarrow \mathbf{G}$ such that the Lie group $($ ker $\varrho)(\mathbb{R})$ is compact and $\Gamma_{0}$ is commensurable with $\varrho(\mathbf{F}(\mathbb{Z}))$, see $[22$, Ch. IX].

Margulis [21] proved the following.

Theorem A (Arithmeticity). Let $\mathbf{G}$ be a connected semisimple $\mathbb{R}$-group so that $\mathbf{G}(\mathbb{R})$ has no compact factors. Let $\Gamma_{0}$ be an irreducible lattice in $\mathbf{G}(\mathbb{R})$. Assume further that $\operatorname{rank}_{\mathbb{R}} \mathbf{G} \geq 2$. Then $\Gamma_{0}$ is arithmetic.

Let $\Gamma_{0}$ and $\mathbf{G}(\mathbb{R})$ be as in Theorem $A$. One may reduce the proof of Theorem A to the case where $\mathbf{G}$ is a group of adjoint type defined over a finitely generated field $\mathrm{L}$ and $\Gamma_{0} \subset \mathbf{G}(\mathrm{L})$ - indeed using local rigidity, one may further assume that $L$ is a number field. The proof of Theorem $A$ is based on applying the following supperrigidity theorem, which was also proved in [21], to representations obtained from different embeddings of $\mathrm{L}$ into local fields.

Theorem B (Superrigidity). Let $\mathbf{G}$ be a connected semisimple $\mathbb{R}$-group. Let $\Gamma_{0}$ be an irreducible lattice in $\mathbf{G}(\mathbb{R})$. Assume further that $\operatorname{rank}_{\mathbb{R}} \mathbf{G} \geq 2$. Let $\mathfrak{l}$ be a local field and let $\mathbf{H}$ be a connected, adjoint, absolutely simple $\mathfrak{l}$-group. Let $\rho: \Gamma_{0} \rightarrow \mathbf{H}(\mathfrak{l})$ be a homomorphism so that $\rho\left(\Gamma_{0}\right)$ is Zariski dense and is not bounded in $\mathbf{H}(\mathfrak{l})$.

Then $\rho$ extends uniquely to a continuous homomorphism $\tilde{\rho}: \mathbf{G}(\mathbb{R}) \rightarrow \mathbf{H}(\mathfrak{l})$.

It follows from the weak approximation theorem that if $\Gamma_{0}$ is an arithmetic group, the index of $\Gamma_{0}$ in $\operatorname{Comm}_{\mathbf{G}(\mathbb{R})}\left(\Gamma_{0}\right)$ is infinite. Margulis proved the converse also holds, see [22, Ch. IX].

A.M. acknowledges support by the NSF. 
Theorem C. Let $\mathbf{G}$ be a connected semisimple $\mathbb{R}$-group so that $\mathbf{G}(\mathbb{R})$ has no compact factors. Let $\Gamma_{0}$ be an irreducible lattice in $\mathbf{G}(\mathbb{R})$. Then $\Gamma_{0}$ is arithmetic if and only if the index of $\Gamma_{0}$ in $\operatorname{Comm}_{\mathbf{G}(\mathbb{R})}\left(\Gamma_{0}\right)$ is infinite.

Supperrigidity and arithmeticity theorems continue to hold for certain rank one Lie groups, namely $\operatorname{Sp}(n, 1)$ and $F_{4}^{-20},[15,5$. However, there are examples of non-arithmetic lattices in $\mathrm{SO}(n, 1)$ for all $n$ and also in $\mathrm{SU}(n, 1)$ for $n=1,2,3$.

Totally geodesic surfaces and arithmeticity. The connected component of the identity in the Lie group $\mathrm{SO}(3,1)$ is isomorphic to

$$
\operatorname{Isom}^{+}\left(\mathbb{H}^{3}\right) \simeq \mathrm{PGL}_{2}(\mathbb{C}) .
$$

Let $M=\mathbb{H}^{3} / \Gamma$ be a closed oriented hyperbolic 3-manifold presented as a quotient of the hyperbolic space by the action of a lattice

$$
\Gamma \subset \mathrm{PGL}_{2}(\mathbb{C}) \text {. }
$$

A totally geodesic surface in $M$ is a proper geodesic immersion of a closed hyperbolic surface into $M$. It is well-known and easy to see that there can be at most countably many totally geodesic surfaces in $M$.

Reid [25] showed that if $\Gamma$ is an arithmetic group, then either $M$ contains no totally geodesic surfaces or it contains infinitely many such surfaces see Theorem $\mathrm{C}$ above. There are also known examples for both of these possibilities, [19]. More recently, it was shown in [9] that a large class of nonarithmetic manifolds contain only finitely many totally geodesic surfaces.

The following theorem is the main result of this paper.

1.1. Theorem. Let $M=\mathbb{H}^{3} / \Gamma$ be a closed hyperbolic 3-manifold. If $M$ contains infinitely many totally geodesic surfaces, then $M$ is arithmetic. That is: $\Gamma$ is an arithmetic lattice.

The statement in Theorem 1.1 answers affirmatively a question asked by A. Reid and C. McMullen, see [23, §8.2] and [6, Qn. 7.6].

This paper is essentially a more detailed version of [20]. More explicitly, several measurability statements were taken for granted in [20], we provide their more or less standard proofs here; moreover, this paper contains a more elaborate version of the proof of Proposition 3.1 when compared to the proof given in [20]. Overall, our goal has been to make this paper as self contained as possible.

Shortly after the appearance of [20, Bader, Fisher, Miller, and Stover [1] proved that if a finite volume hyperbolic $n$-manifold, $\mathbb{H}^{n} / \Gamma$, contains infinitely many maximal totally geodesic subspaces of dimension at least 2 , then $\Gamma$ is arithmetic - Theorem 1.1 is a special case. Their proof and ours both use a superrigidity theorem to prove arithmeticity, but the superrigidity theorems and their proofs are quite different. 
In view of Theorem $\mathrm{C}$, we get the following from Theorem 1.1, If $M=$ $\mathbb{H}^{3} / \Gamma$ is a closed hyperbolic 3 -manifold which contains infinitely many totally geodesic surfaces, the index of $\Gamma$ in its commensurator is infinite.

As was mentioned above the arithmeticity theorem for irreducible lattices in higher rank Lie groups was proved using the superrigidity Theorem B. Similarly, Theorem 1.1 follows from Theorem 1.2 below which is a rigidity type result.

A rigidity theorem. Let $\mathrm{L}$ be the number field and $\mathbf{G}$ the connected, semisimple L-group of adjoint type associated to $\Gamma$, see $₫ 2$ also [22, 19]. In particular, $\mathrm{L} \subset \mathbb{R}, \Gamma \subset \mathbf{G}(\mathrm{L})$, and $\mathbf{G}$ is $\mathbb{R}$-isomorphic to $\mathrm{PO}(3,1)$ - the connected component of the identity in the Lie group $\mathbf{G}(\mathbb{R})$ is isomorphic to $\mathrm{PGL}_{2}(\mathbb{C})$.

Let $\mathcal{S}$ denote the set of places of $\mathrm{L}$. For every $v \in \mathcal{S}$, let $\mathrm{L}_{v}$ be the completion of $\mathrm{L}$ at $v$ and let $\Sigma_{v}$ be the set of Galois embeddings $\sigma: \mathrm{L} \rightarrow \mathrm{L}_{v}$.

For any $v \in \mathcal{S}$ and any $\sigma \in \Sigma_{v}$, we let ${ }^{\sigma} \mathbf{G}$ denote the algebraic group defined by applying $\sigma$ to the equations of $\mathbf{G}$. Let $v \in \mathcal{S}$ and $\sigma \in \Sigma_{v}$, then $\sigma(\Gamma) \subset{ }^{\sigma} \mathbf{G}$ is Zariski dense.

1.2. Theorem. Let $M=\mathbb{H}^{3} / \Gamma$ be a closed hyperbolic 3-manifold. Assume further that $M$ contains infinitely many totally geodesic surfaces. Let $\mathrm{L}$ and $\mathbf{G}$ be as above.

If $v \in \mathcal{S}$ and $\sigma \in \Sigma_{v}$ are so that $\sigma(\Gamma) \subset{ }^{\sigma} \mathbf{G}\left(\mathrm{L}_{v}\right)$ is unbounded, then $\sigma$ extends to a continuous homomorphism from $\mathbf{G}(\mathbb{R})$ to ${ }^{\sigma} \mathbf{G}\left(\mathrm{L}_{v}\right)$.

Theorem 1.1 follows from Theorem 1.2. We will recall the argument from [21] in 3.8 - indeed the group $\mathbf{F}$ in the definition of an arithmetic group is the Zariski closure of $\Gamma$ in the restriction of scalars group $R_{L / \mathbb{Q}}(G)$, see also [22, Ch. IX].

The proof of Theorem 1.2 is based on the study of certain $\Gamma$-equivariant measurable maps from $\partial \mathbb{H}^{3}=\mathbb{S}^{2}$ into projective spaces — equivariant maps of this kind also play a pivotal role in the proof of the strong rigidity theorem by Mostow and the proof of the superrigidity theorem by Margulis.

Indeed the proof in [21] is based on showing that an a priori only measurable boundary map agrees with a rational map almost surely; this rationality is then used to find the desired continuous extension. Our strategy here is to show that if $M$ contains infinitely many totally geodesic surfaces, a certain $\Gamma$-equivariant measurable map on $\mathbb{S}^{2}$ is almost surely rational, see Proposition 3.1. In 3.8 we use Proposition 3.1 to complete the proof of Theorem 1.2 , see [21].

We end the introduction by mentioning that in this paper the discussion is restricted to the case of closed hyperbolic 3-manifolds; however, our method extends to the case of finite volume hyperbolic 3-manifolds. Indeed our argument rests upon investigating certain properties of a cocycle which will be introduced in 95 . The extension to finite volume hyperbolic 3-manifolds requires some estimates for the growth rate of this cocycle. The desired 
estimates may be obtained using a similar, and simpler, version of systems of inequalities in [7].

Acknowledgement. We would like to thank D. Fisher, C. McMullen, $\mathrm{H}$. Oh, and A. Reid for their helpful comments on earlier versions of this paper.

\section{Preliminaries AND NOtATiOn}

Let $G=\mathrm{PGL}_{2}(\mathbb{C})$ and let $\Gamma \subset G$ be a lattice. Let $X=G / \Gamma$ and let $\operatorname{vol}_{X}$ (or simply vol) denote the $G$-invariant probability measure on $X$. We let $\pi$ denote the natural projection from $G$ to $X$. Also let $K=\mathrm{SU}(2) /\{ \pm I\}$.

We let $H=\mathrm{PGL}_{2}(\mathbb{R})$. For every $t \in \mathbb{R}$, let

$$
a_{t}=\left(\begin{array}{cc}
e^{t / 2} & 0 \\
0 & e^{-t / 2}
\end{array}\right)
$$

note that $a_{t} \in H$ for all $t \in \mathbb{R}$. For every $\theta \in[0,2 \pi], r_{\theta} \in \mathrm{PGL}_{2}(\mathbb{R})$ denotes the rotation with angle $\theta$.

The bundle of oriented frames over $\mathbb{H}^{3}=K \backslash G$ may be identified with $G$. The left action of $\left\{a_{t}: t \in \mathbb{R}\right\}$ on $G$ and $G / \Gamma$ induces the frame flow on the frame bundles of $\mathbb{H}^{3}$ and $M$, respectively. For any $g \in G$ the image of $H g$ in $\mathbb{H}^{3}$ is a geodesic embedding of $\mathbb{H}^{2}$ into $\mathbb{H}^{3}$. In this setup, a totally geodesic surface in $M=K \backslash G / \Gamma$ lifts to a closed orbit of $H$ in $X$.

2.1. The number field $L$ and the $L$-group $G$. Since $\Gamma$ is finitely generated, there exists a finitely generated field $L$ and a connected, semisimple L-group $\mathbf{G}$ of adjoint type so that $\mathrm{L} \subset \mathbb{R}, \Gamma \subset \mathbf{G}(\mathrm{L})$, and $\mathbf{G}$ is $\mathbb{R}$-isomorphic to $\mathrm{PO}(3,1)$. In view of local rigidity of $\Gamma,[13$, Thm. 0.11], $\mathrm{L}$ is indeed a number field, see also [26, 27, 28]. We will refer to the pair $(\mathbf{L}, \mathbf{G})$ as the number field and the group associated to $\Gamma$, see [22, 19].

Let $\mathcal{S}$ denote the set of places of $\mathrm{L}$. For every $v \in \mathcal{S}$, let $\mathrm{L}_{v}$ be the completion of $\mathrm{L}$ at $v$ and let $\Sigma_{v}$ be the set of Galois embeddings $\sigma: \mathrm{L} \rightarrow \mathrm{L}_{v}$.

With this notation we let $\left(v_{0}\right.$, id) be the pair which gives rise to the lattice $\Gamma$ in $G$ - recall that the connected component of the identity in the Lie group $\mathbf{G}(\mathbb{R})$ is isomorphic to $\mathrm{PGL}_{2}(\mathbb{C})$.

For any $v \in \mathcal{S}$ and any $\sigma \in \Sigma_{v}$, we let ${ }^{\sigma} \mathbf{G}$ denote the $\sigma(\mathrm{L})$-group defined by applying $\sigma$ to the coefficients of the defining equations of G. Let $v \in \mathcal{S}$ and $\sigma \in \Sigma_{v}$, then $\sigma(\Gamma) \subset{ }^{\sigma} \mathbf{G}$ is Zariski dense.

Note that $\mathbf{G}$ is isomorphic to $\mathrm{PGL}_{2} \times \mathrm{PGL}_{2}$ over $\mathbb{C}$. More generally, for every $v \in \mathcal{S}$, there exists an extension $\mathfrak{l}_{v} / \mathbf{L}_{v}$ of degree at most 2 so that ${ }^{\sigma} \mathbf{G}$ splits over $\mathfrak{l}_{v}$. That is:

$$
{ }^{\sigma} \mathbf{G} \text { is isomorphic to } \mathrm{PGL}_{2} \times \mathrm{PGL}_{2} \text { over } \mathfrak{l}_{v} \text {. }
$$

Let $\Delta \subset \Gamma$ be a non-elementary Fuchsian group; in the above notation, we have the following. Let $\mathbf{H}_{\Delta}$ be the Zariski closure of $\Delta$ in $\mathbf{G}$. Then the Lie group $\mathbf{H}_{\Delta}(\mathbb{R})$ is locally isomorphic to $\mathrm{SL}_{2}(\mathbb{R})$. 
For every $v \in \mathcal{S}$ and every $\sigma \in \Sigma_{v}$, let ${ }^{\sigma} \mathbf{H}_{\Delta} \subset{ }^{\sigma} \mathbf{G}$ denote the $\sigma(\mathrm{L})$-group obtained by applying $\sigma$ to the defining coefficients of $\mathbf{H}_{\Delta}$ - note that ${ }^{\sigma} \mathbf{H}_{\Delta}$ is the Zariski closure of $\sigma(\Delta)$ in ${ }^{\sigma} \mathbf{G}$.

2.2. Lemma. Let $\Delta \subset \Gamma$ be a non-elementary Fuchsian group. Assume that $\sigma(\Delta)$ is unbounded in ${ }^{\sigma} \mathbf{G}\left(\mathfrak{l}_{v}\right)=\mathrm{PGL}_{2}\left(\mathfrak{l}_{v}\right) \times \mathrm{PGL}_{2}\left(\mathfrak{l}_{v}\right)$. Then there exists some $g \in \mathrm{PGL}_{2}\left(\mathfrak{l}_{v}\right)$ so that

$$
{ }^{\sigma} \mathbf{H}\left(\mathfrak{l}_{v}\right) \cap\left\{\left(h, g h g^{-1}\right): h \in \mathrm{PGL}_{2}\left(\mathfrak{l}_{v}\right)\right\}
$$

is a subgroup of index at most 8 in ${ }^{\sigma} \mathbf{H}\left(\mathfrak{l}_{v}\right)$.

Proof. The group ${ }^{\sigma} \mathbf{H}$ is isogeneous to $\mathrm{SL}_{2}$, in particular, it is a proper algebraic subgroup of ${ }^{\sigma} \mathbf{G}$. Moreover, ${ }^{\sigma} \mathbf{H}$ intersects each factor of ${ }^{\sigma} \mathbf{G}$ trivially. Indeed, ${ }^{\sigma} \mathbf{G}$ has trivial center; therefore, if ${ }^{\sigma} \mathbf{H}$ intersects a factor nontrivially, then this intersection is normal in ${ }^{\sigma} \mathbf{H}$ which implies that ${ }^{\sigma} \mathbf{H} \triangleleft^{\sigma} \mathbf{G}$. Now since $\mathbf{H}(\mathrm{L})$ and $\mathbf{G}(\mathrm{L})$ are Zariski dense in $\mathbf{H}$ and $\mathbf{G}$, respectively, we get that $\mathbf{H} \triangleleft \mathbf{G}$; this is a contradiction.

In consequence, there exists an $\mathfrak{l}_{v}$-group $\mathbf{L}$ which is an $\mathfrak{l}_{v}$-form of $\mathrm{SL}_{2}$, i.e., either arising from a division algebra or $\mathrm{SL}_{2}$, and an $\mathfrak{l}_{v}$-isogeny $\varphi: \mathbf{L} \rightarrow{ }^{\sigma} \mathbf{H}$, so that ${ }^{\sigma} \mathbf{H}\left(\mathfrak{l}_{v}\right)$ contains $\varphi\left(\mathbf{L}\left(\mathfrak{l}_{v}\right)\right)$ as a subgroup of finite index. We claim $\mathbf{L}$ is indeed $\mathrm{SL}_{2}$. The group $\sigma(\Delta)$ is unbounded, therefore, the group ${ }^{\sigma} \mathbf{H}\left(\mathfrak{l}_{v}\right)$ is unbounded. Hence, ${ }^{\sigma} \mathbf{H}$ is $\mathfrak{k}_{v}$-isotropic, this implies the claim.

Since ${ }^{\sigma} \mathbf{H}$ is a group of type $A_{1}$, the isogeny $\varphi$ is inner, i.e., it is induced by conjugation with an element $g \in \mathrm{PGL}_{2}\left(\mathfrak{l}_{v}\right)$.

The claim regarding the index follows as $\left[\mathrm{PGL}_{2}(\mathfrak{l}): \mathrm{SL}_{2}(\mathfrak{l})\right] \leq 8$ for any local field $\mathfrak{l}$ of characteristic zero.

2.3. Lemmas from hyperbolic geometry. In this section we will recrod some basic facts from hyperbolic geometry to be used in the sequel. Let us begin by recalling the hyperbolic law of cosines. Let $p q r$ be a hyperbolic triangle and let $\theta$ be the angle opposite to the edge $\overline{q r}$. Then

$$
\cosh (\overline{q r})=\cosh (\overline{p q}) \cosh (\overline{p r})-\cos (\theta) \sinh (\overline{p q}) \sinh (\overline{p r})
$$

Let $\left(\mathcal{T}, \mathrm{d}_{\mathcal{T}}\right)$ denote either a regular tree equipped with the usual path metric or a hyperbolic space equipped with the hyperbolic metric. We fix a base point $o \in \mathcal{T}$.

2.4. Lemma. Let $\left\{p_{n}: n=0,1, \ldots\right\} \subset \mathcal{T}$ with $p_{0}=o$. Assume that there exist some $L_{1}, L_{2}, N_{0}>1$ so that $\mathrm{d}_{\mathcal{T}}\left(p_{n}, p_{(n+1)}\right) \leq L_{1}$ for all $n$ and

$$
\mathrm{d}_{\mathcal{T}}\left(p_{n}, o\right) \geq n / L_{2} \text { for all } n>N_{0} .
$$

Then there exists a unique geodesic $\xi=\left\{\xi_{t}: t \in \mathbb{R}\right\} \subset \mathcal{T}$ with $\xi_{0}=o$ so that

$$
p_{n} \rightarrow \xi_{\infty} \in \partial \mathcal{T} \text {. }
$$

Moreover, for every $\varepsilon>0$ there exists some $N=N\left(L_{1}, L_{2}, N_{0}, \varepsilon\right)$ so that for all $n>N$ we have $\mathrm{d}_{\mathcal{T}}\left(p_{n}, \xi\right) \leq \varepsilon n$.

We will also need the following lemma; the proof of this lemma uses Lemma 2.4 and Egorov's theorem. 
2.5. Lemma. Let $(\Theta, \vartheta)$ be a Borel probability space. Let $\psi: \Theta \rightarrow \partial \mathcal{T}$ and $u: \mathbb{Z}^{\geq 0} \times \Theta \rightarrow \mathcal{T}$ be two Borel maps satisfying the following.

(1) $\vartheta\left(\psi^{-1}\{p\}\right)=0$ for every $p \in \partial \mathcal{T}$.

(2) $u(0, \theta)=$ o for a.e. $\theta \in \Theta$.

(3) There exists some $L_{1} \geq 1$ so that for a.e. $\theta \in \Theta$ we have

$$
\mathrm{d}_{\mathcal{T}}\left(u(n, \theta), u(n+1, \theta) \leq L_{1} \quad \text { for all } n .\right.
$$

(4) There exists some $L_{2} \geq 1$ and for a.e. $\theta \in \Theta$ there exists some $N_{\theta}$ so that

$$
\mathrm{d}_{\mathcal{T}}\left(u(n, \theta), u(n+1, \theta) \geq n / L_{2} \text { for all } n>N_{\theta} .\right.
$$

In particular, by Lemma 2.4 we have $\{u(n, \theta)\}$ converges to a point in $\partial \mathcal{T}$ for a.e. $\theta \in \Theta$. Assume further that

$$
u(n, \theta) \rightarrow \psi(\theta) \text { for a.e. } \theta \in \Theta .
$$

Let $\left\{\xi_{t}\right\} \subset \mathcal{T}$ be any geodesic with $\xi_{0}=o$. There exists some $c^{\prime}=c^{\prime}\left(\psi, u, L_{1}, L_{2}\right)$ so that for all $t \in \mathbb{R}$ and all $n \in \mathbb{N}$ we have

$$
\int_{\Theta} \mathrm{d}_{\mathcal{T}}\left(u(n, \theta), \xi_{t}\right) \mathrm{d} \vartheta(\theta)>t+\frac{n}{4 L_{2}}-c^{\prime} .
$$

Proof. We explicate the proof when $\mathcal{T}$ is a hyperbolic space, the proof in the case of a tree is a simple modification.

For any $\theta \in \Theta$, let $q_{\theta}=u(n, \theta)$. By the hyperbolic law of cosines, (2.3), we have

$$
\cosh \left(\overline{q_{\theta} \xi_{t}}\right)=\cosh \left(\overline{o \xi_{t}}\right) \cosh \left(\overline{o q_{\theta}}\right)-\cos (\alpha) \sinh \left(\overline{o \xi_{t}}\right) \sinh \left(\overline{o q_{\theta}}\right)
$$

where $\alpha$ is the angle between $\overline{o \xi_{t}}$ and $\overline{o q_{\theta}}$.

In view of our assumption (1) and the compactness of $\partial \mathcal{T}$ for every $\varepsilon>0$ there exists some $\delta>0$ so that for every $p \in \partial \mathcal{T}$ we have

$$
\vartheta\left(\psi^{-1}\left(\mathrm{~N}_{\delta}(p)\right)\right)<\varepsilon / 2 .
$$

Fix some $0<\varepsilon<1 / 8 L_{1} L_{2}$. In view of Egorov's theorem, Lemma 2.4 and (2.4), there exists $\Theta^{\prime} \subset \Theta$ with $\vartheta\left(\Theta^{\prime}\right)>1-\varepsilon / 2$ and some $N_{0} \geq 1$ so that for all $\theta \in \Theta^{\prime}$ and all $n>N_{0}$ we have

$$
\mathrm{d}_{\mathcal{T}}(u(n, \theta), o) \geq n / 2 L_{2} .
$$

Let $\mathrm{E}:=\left\{\theta \in \Theta^{\prime}: \psi(\theta) \notin \mathrm{N}_{\delta}\left(\xi_{\infty}\right)\right\}$ where $\xi_{\infty}=\lim _{t \rightarrow \infty} \xi_{t} \in \partial \mathcal{T}$. Then thanks to (2.6) and $\vartheta\left(\Theta^{\prime}\right)>1-\varepsilon / 2$ we have $\vartheta(\mathrm{E})>1-\varepsilon$.

Now using (2.5) and (2.7), we conclude that

$$
\mathrm{d} \mathcal{T}\left(q_{\theta}, \xi_{t}\right)>t+\frac{n}{2 L_{2}}-O_{\delta}(1)
$$

for all $\theta \in \mathrm{E}$ and all $n>N_{0}$.

Note also that by our assumption (3), we have $\mathrm{d}_{\mathcal{T}}(u(n, \theta), o) \leq L_{1} n$ for all $\theta \in \Theta$. In particular, we get that

$$
\mathrm{d}_{\mathcal{T}}\left(q_{\theta}, \xi_{t}\right) \geq t-L_{1} n
$$

for all $\theta \in \Theta$ and all $n \in \mathbb{N}$. 
Now, splitting the integral over $\mathrm{E}$ and its compliment and using the estimates in (2.8) and (2.9), we obtain the following:

$$
\begin{aligned}
\int_{\Theta} \mathrm{d} \mathcal{T}\left(q_{\theta}, \xi_{t}\right) \mathrm{d} \vartheta(\theta) & >(1-\varepsilon)\left(t+\frac{n}{2 L_{2}}-O_{\delta}(1)\right)+\left(t-L_{1} n\right) \varepsilon \\
\varepsilon<1 / 4 \rightsquigarrow & >t+\frac{n}{4 L_{2}}+\left(\frac{n}{8 L_{2}}-L_{1} \varepsilon n\right)-O_{\delta}(1) \\
\varepsilon<1 / 8 L_{1} L_{2} \rightsquigarrow & >t+\frac{n}{4 L_{2}}-O_{\delta}(1) .
\end{aligned}
$$

This completes the proof if we let $c^{\prime}=O_{\delta}(1)$.

It is worth mentioning that the above lemmas hold for any proper, complete, $\operatorname{CAT}(-1)$ space.

2.6. Action of $\Gamma$ on varieties. Let $\mathcal{C}$ be the space of circles in $\mathbb{S}^{2}=\partial \mathbb{H}^{3}$; the space $\mathcal{C}$ is equipped with a natural $\operatorname{PGL}_{2}(\mathbb{C})$-invariant measure $\sigma$. Let $\mathrm{m}$ denote the Lebesgue measure on $\mathbb{S}^{2}$. For every $C \in \mathcal{C}$, let $\mathrm{m}_{C}$ be the Lebesgue measure on $C$.

Here and in what follows, by a non-atomic measure we mean a measure without any atoms.

The following general lemma will be used in the sequel.

2.7. Lemma. Let $\mathfrak{l}$ be a local field. Let $\mathbf{H}$ be a connected $\mathfrak{l}$-group which acts $\mathfrak{l}$-rationally on an irreducible $\mathfrak{l}$-variety $\mathbf{V}$. Assume further that $\mathbf{H}$ acts transitively on $\mathbf{V}$. Let $\rho: \Gamma \rightarrow \mathbf{H}(\mathfrak{l})$ be a homomorphism so that $\rho(\Gamma)$ is Zariski dense in $\mathbf{H}$ and let $\Psi: \mathbb{S}^{2} \rightarrow \mathbf{V}(\mathfrak{l})$ be a $\Gamma$-equivariant measurable map. Then

(1) $\mathrm{m}\left(\left\{\xi \in \mathbb{S}^{2}: \Psi(\xi) \in \mathbf{W}(\mathfrak{l})\right\}\right)=0$ for any proper subvariety $\mathbf{W} \subset \mathbf{V}$.

(2) For $\sigma$-a.e. $C \in \mathcal{C}$ and every $p \in \mathbf{V}(\mathfrak{l})$ we have

$$
\mathrm{m}_{C}(\{\xi \in C: \Psi(\xi)=p\})=0 .
$$

Proof. Part (1) is well-known, see [14, Lemma 4.2] also [22, Ch. VI, Lemma 3.10] and [12.

We prove part (2). Let $\mathcal{A}=\left\{\left(C, \xi, \xi^{\prime}\right) \in \mathcal{C} \times \mathbb{S}^{2} \times \mathbb{S}^{2}: \xi, \xi^{\prime} \in C\right\}$. Equip $\mathcal{A}$ with the natural measure arising from the $G$-invariant measure $\sigma$ on $\mathcal{C}$ and the measure $\mathrm{m}_{C}$ on $C \in \mathcal{C}$.

Define $\Phi: \mathcal{A} \rightarrow \mathbf{V}(\mathfrak{l}) \times \mathbf{V}(\mathfrak{l})$ by $\Phi\left(C, \xi, \xi^{\prime}\right)=\left(\Psi(\xi), \Psi\left(\xi^{\prime}\right)\right)$. Assume the claim in part (2) fails. Then

$$
\mathcal{B}:=\Phi^{-1}(\{(p, p): p \in \mathbf{V}(\mathfrak{l})\}) \subset \mathcal{A}
$$

has positive measure.

In view of Fubini's theorem then the projection of $\mathcal{B}$ onto $\mathbb{S}^{2} \times \mathbb{S}^{2}$ contains a positive measure subset. That is: there exists a positive measure subset of $\mathbb{S}^{2} \times \mathbb{S}^{2}$ which gets mapped into $\{(p, p): p \in \mathbf{V}(\mathfrak{l})\}$. This implies that $\Psi$ maps a positive measure subset of $\mathbb{S}^{2}$ to a point, which contradicts part (1).

For any compact subset $E \subset \mathbb{H}^{3} / \Gamma$, let $\mathcal{C}_{E}=\{C \in \mathcal{C}$ : the convex hull of $C$ intersects $E$ nontrivially . 
2.8. Lemma. Let the notation be as in Lemma 2.7. Further, assume that $\mathbf{V}(\mathfrak{l})$ is compact and that it is equipped with a metric. For every $p \in \mathbf{V}(\mathfrak{l})$ and every $r>0$, let $\mathrm{N}_{r}(p)$ denote the open ball of radius $r$ in this metric.

Let $E \subset \mathbb{H}^{3} / \Gamma$ be a compact subset with positive measure. For every $\varepsilon>0$ there exists a compact subset $\mathcal{C}_{E, \varepsilon} \subset \mathcal{C}_{E}$ with $\sigma\left(\mathcal{C}_{E} \backslash \mathcal{C}_{E, \varepsilon}\right) \ll_{E} \varepsilon^{32} \sigma\left(\mathcal{C}_{E}\right)$ and some $\delta>0$ with the following property. For every $C \in \mathcal{C}_{E, \varepsilon}$ and every $p \in \mathbf{V}(\mathfrak{l})$ we have

$$
\mathrm{m}_{C}\left(\left\{\xi \in C: \Psi(\xi) \in \mathrm{N}_{\delta}(p)\right\}\right)<\varepsilon \mathrm{m}_{C}(C) .
$$

Proof. First note that $\mathcal{C}_{E}$ is a compact subset of $\mathcal{C}$ with $\sigma\left(\mathcal{C}_{E}\right)>0$. Note also that, up to a null set, we may identify the space of circles $\mathcal{C}$ with $\partial \mathbb{H}^{3} \times(0,1]$.

Therefore, ignoring a possible null subset, there exist $r, r^{\prime}>0$ so that

$$
\mathcal{C}_{E} \subset \partial \mathbb{H}^{3} \times[r, 1]=: \mathcal{C}_{r}
$$

and $\mathrm{m}\left(\mathcal{C}_{E}\right) \geq r^{\prime} \mathrm{m}\left(\mathcal{C}_{r}\right)$.

By Lusin's theorem, there exists a compact subset $D \subset \partial \mathbb{H}^{3}$ with $\mathrm{m}(D)>$ $1-\varepsilon^{64}$ so that $\left.\Psi\right|_{D}$ is continuous.

For every $\xi \in \partial \mathbb{H}^{3}$, set

$$
I_{\xi}:=\left\{t \in[r, 1]: \mathrm{m}_{C_{t}(\xi)}\left(C_{t}(\xi) \cap D\right)<\left(1-\varepsilon^{32}\right) \mathrm{m}_{C_{t}(\xi)}\left(C_{t}(\xi)\right)\right\}
$$

where $C_{t}(\xi)$ is the circle with radius $t$ centered at $\xi$. By Fubini's theorem, we have: $\left|I_{\xi}\right| \ll_{r} \varepsilon^{32}$ for every $\xi \in \partial \mathbb{H}^{3}$. Therefore,

$$
\sigma\left(\left\{C \in \mathcal{C}_{r}: \mathrm{m}_{C}(C \cap D)<\left(1-\varepsilon^{32}\right) \mathrm{m}_{C}(C)\right\}\right) \ll_{r} \varepsilon^{32} .
$$

Let $\hat{\mathcal{C}}_{E, \varepsilon}=\left\{C \in \mathcal{C}_{E}: \mathrm{m}_{C}(C \cap D) \geq\left(1-\varepsilon^{32}\right) \mathrm{m}_{C}(C)\right\}$. In view of the above estimate and since $\mathcal{C}_{E} \subset \mathcal{C}_{r}$, we have $\sigma\left(\mathcal{C}_{E} \backslash \hat{\mathcal{C}}_{E, \varepsilon}\right) \ll_{r, r^{\prime}} \varepsilon^{32}$.

Let $\mathcal{C}^{\prime} \subset \mathcal{C}$ be the conull subset where Lemma 2.7(2) holds true. Let $\mathcal{C}_{E, \varepsilon}$ be a compact subset of $\hat{\mathcal{C}}_{E, \varepsilon} \cap \mathcal{C}^{\prime}$ so that $\sigma\left(\mathcal{C}_{E} \backslash \mathcal{C}_{E, \varepsilon}\right) \ll_{r, r^{\prime}} \varepsilon^{32} \sigma\left(\mathcal{C}_{E}\right)$. In particular, $\mathcal{C}_{E}$ satisfies the first claim in the lemma. We now verify that it also satisfies the second claim.

Assume contrary to the claim that for every $n$ there is some $p_{n} \in \mathbf{V}(\mathfrak{l})$ and some $C_{n} \in \mathcal{C}_{E, \varepsilon}$ so that

$$
\mathrm{m}_{C_{n}}\left(\left\{\xi \in C_{n}: \Psi(\xi) \in \mathrm{N}_{1 / n}\left(p_{n}\right)\right\}\right)>\varepsilon \mathrm{m}_{C_{n}}\left(C_{n}\right) .
$$

Passing to a subsequence, if necessary, we assume that $C_{n} \rightarrow C \in \mathcal{C}_{E, \varepsilon}$ and $p_{n} \rightarrow p \in \mathbf{V}(\mathfrak{l})$ - recall that $\mathbf{V}(\mathfrak{l})$ is compact.

For each $n$, let $C_{n}^{\prime}:=\left\{\xi \in C_{n}: \Psi(\xi) \in \mathrm{N}_{1 / n}\left(p_{n}\right)\right\} \cap D$. In view of the fact that $\mathcal{C}_{E, \varepsilon} \subset \hat{\mathcal{C}}_{E, \varepsilon}$ and using (2.10), we get that

$$
\mathrm{m}_{C_{n}}\left(C_{n}^{\prime}\right) \geq \varepsilon \mathrm{m}_{C_{n}}\left(C_{n}\right) / 2 .
$$

Let $C^{\prime}:=\lim \sup C_{n}^{\prime}$; then $C^{\prime} \subset C \cap D$ and $\mathrm{m}_{C}\left(C^{\prime}\right) \geq \varepsilon \mathrm{m}_{C}(C) / 2$. Moreover, for every $\xi \in C^{\prime}$ there exist some $\xi_{n} \rightarrow \xi$ with $\xi_{n} \in C_{n}^{\prime}$. Since $\Psi$ is continuous on $D$, we get that $\Psi(\xi)=p$, i.e., $\Psi\left(C^{\prime}\right)=p$. This contradicts the fact that $C^{\prime} \in \mathcal{C}_{E, \varepsilon} \subset \mathcal{C}^{\prime}$ and finishes the proof. 


\section{A T-Equivariant CIRCle PRESERVING MAP}

In this section we state one of the main results of this paper, Proposition 3.1. We then complete the proofs of Theorem 1.2 and Theorem 1.1 using Proposition 3.1.

Let the notation be as in 92.1 In particular, $\mathrm{L}$ is a number field and $\mathbf{G}$ is an L-group. For every $v \in \mathcal{S}$ and $\sigma \in \Sigma_{v}$, let $\mathfrak{l}_{v}$ denote $\mathbb{C}$ if $v$ is an Archimedean place, and an extension of degree at most 2 of $\mathrm{L}_{v}$ so that ${ }^{\sigma} \mathbf{G}$ is $\mathfrak{l}_{v}$-split if $v$ is a non-Archimedean place. Recall from (2.2) that

${ }^{\sigma} \mathbf{G}$ is $\mathfrak{l}_{v}$-isomorphic to $\mathrm{PGL}_{2} \times \mathrm{PGL}_{2}$.

Let $B_{v}$ denote the group of upper triangular matrices in $\mathrm{PGL}_{2}\left(\mathfrak{l}_{v}\right)$. For every $g \in \mathrm{PGL}_{2}\left(\mathfrak{l}_{v}\right)$ define $\mathfrak{C}_{g}$ to be the image of

$$
\left\{\left(h, g h g^{-1}\right): h \in \mathrm{PGL}_{2}\left(\mathfrak{l}_{v}\right)\right\}
$$

in $B_{v} \backslash \mathrm{PGL}_{2}\left(\mathfrak{l}_{v}\right) \times B_{v} \backslash \mathrm{PGL}_{2}\left(\mathfrak{l}_{v}\right)=\mathbb{P l}_{v} \times \mathbb{P l}_{v}$.

Note that for every $g, \mathfrak{C}_{g}$ is the graph of the linear fractional transformation $g: \mathbb{P l}_{v} \rightarrow \mathbb{P l}_{v}$.

Recall from 22.6 that $\mathcal{C}$ denotes the space of circles in $\mathbb{S}^{2}=\partial \mathbb{H}^{3}$; the space $\mathcal{C}$ is equipped with a natural $\mathrm{PGL}_{2}(\mathbb{C})$-invariant measure $\sigma$. Recall also that $\mathrm{m}$ denotes the Lebesgue measure on $\mathbb{S}^{2}$, and that for every $C \in \mathcal{C}$, $\mathrm{m}_{C}$ denotes the Lebesgue measure on $C$.

3.1. Proposition. Assume $X=G / \Gamma$ contains infinitely many closed $H$ orbits. Let $\mathfrak{l}_{v}$ and $\mathbf{G}$ be as above and assume that

$$
\sigma(\Gamma) \subset \mathrm{PGL}_{2}\left(\mathfrak{l}_{v}\right) \times \mathrm{PGL}_{2}\left(\mathfrak{l}_{v}\right) \text { is unbounded } .
$$

There exists a $\Gamma$-equivariant measurable map

$$
\Psi: \mathbb{S}^{2} \rightarrow \mathbb{P l}_{v} \times \mathbb{P l}_{v}
$$

with the following properties.

(1) For a.e. $C \in \mathcal{C}$ we have $\left(\left.\Psi\right|_{C}\right)_{*} \mathrm{~m}_{C}$ is non-atomic.

(2) For a.e. $C \in \mathcal{C}$ there exists some $g_{C} \in \mathrm{PGL}_{2}\left(\mathfrak{l}_{v}\right)$ so that the essential image of $\left.\Psi\right|_{C}$ is contained in $\mathfrak{C}_{g_{C}}$.

This proposition will be proved in 86 , Our goal in this section is to complete the proofs of Theorem 1.2 and Theorem 1.1 using Proposition 3.1.

In the sequel by an inversion of a circle $C$ we mean a linear fractional transformation on $C$ of order 2 where $C$ is identified with $\mathbb{P R}$; similarly we define an inversion of $\mathfrak{C}_{g}$.

3.2. Lemma. Let $\varphi: C \rightarrow \mathfrak{C}_{g}$ be a Borel measurable map so that the essential image of $\varphi$ has at least three points. Let $\mathcal{I}$ be a subset of inversions on $C$ which generates $\mathrm{PSL}_{2}(\mathbb{R})$. Assume further that there exists a Borel map $f$ from $\mathcal{I}$ into the set of inversions on $\mathfrak{C}_{g}$ which satisfies the following:

$$
\text { for any } \iota \in \mathcal{I}, \varphi \circ \iota=f(\iota) \circ \varphi \quad \mathrm{m}_{C} \text {-a.e. on } C \text {. }
$$

Then $f$ extends to a continuous homomorphism from $\mathrm{PSL}_{2}(\mathbb{R})$ into $\mathrm{PGL}_{2}\left(\mathfrak{l}_{v}\right)$. 
Proof. First note that since the essential image of $\varphi$ has at least three points, any linear fractional transformation on $\mathfrak{C}_{g}$ is uniquely determined by its restriction to the essential image of $\varphi$.

In view of this and (3.1) the map $\iota_{1} \circ \cdots \circ \iota_{n} \mapsto f\left(\iota_{1}\right) \circ \cdots \circ f\left(\iota_{n}\right)$ is a well-defined measurable homomorphism from the group generated by $\mathcal{I}$ into $\mathrm{PGL}_{2}\left(\mathfrak{l}_{v}\right)$.

The claim follows from this as $\mathcal{I}$ generates $\mathrm{PSL}_{2}(\mathbb{R})$ and any measurable homomorphism is continuous, see e.g. [22, Ch. VII, Lemma 1.4].

Let $\left(\xi, \xi^{\prime}\right) \in \mathbb{S}^{2} \times \mathbb{S}^{2}$, with $\xi \neq \xi^{\prime}$. We let $C_{t}\left(\xi, \xi^{\prime}\right), t \in I_{\xi, \xi^{\prime}} \subset \mathbb{R}$ denote the one parameter family of circles in $\mathbb{S}^{2}$ passing through $\xi$ and $\xi^{\prime}$.

Given a triple $\left(\xi, \xi^{\prime}, C\right) \in \mathbb{S}^{2} \times \mathbb{S}^{2} \times \mathcal{C}$ we say $\left\{\xi, \xi^{\prime}\right\}$ and $C$ are linked if $\xi$ and $\xi^{\prime}$ belong to different connected components of $\mathbb{S}^{2} \backslash C$. For every circle $C \in \mathcal{C}$, let

$$
E_{C} \subset \mathbb{S}^{2} \times \mathbb{S}^{2}
$$

denote the set of $\left(\xi, \xi^{\prime}\right) \in \mathbb{S}^{2} \times \mathbb{S}^{2}$ so that $\left\{\xi, \xi^{\prime}\right\}$ and $C$ are linked.

3.3. Lemma. Let $C \in \mathcal{C}$ and $\left(\xi, \xi^{\prime}\right) \in \mathbb{S}^{2} \times \mathbb{S}^{2}$; assume that $\left\{\xi, \xi^{\prime}\right\}$ and $C$ are linked. Then the one parameter family $\left\{C_{t}\left(\xi, \xi^{\prime}\right): t \in I_{\xi, \xi^{\prime}}\right\}$ defines an inversion on the circle $C$.

Proof. Define $\iota_{\xi, \xi^{\prime}}$ on $C$ as follows. Let $p \in C$, there exists a unique circle $C^{\prime} \in C_{t}\left(\xi, \xi^{\prime}\right)$ which passes through $\xi, \xi^{\prime}, p$. Then $C^{\prime} \cap C=\{p, q\}$. Let $\iota_{\xi, \xi^{\prime}}(p)=q$ - note that $\iota_{\xi, \xi^{\prime}}$ has order two. The map $\iota_{\xi, \xi^{\prime}}$ is an inversion on $C$. This could be seen as follows: we may assume $C$ is the unit circle in the plane, $\xi^{\prime}=\infty$ and $\xi=(0, b)$ for some $0 \leq b<1$. Let pr denote the stereographic projection of $C$ onto the line $\{y=b\} \cup\{\infty\}$. Then $\operatorname{pr}(0,1)=\infty$ and $\iota_{\xi, \xi^{\prime}}(0,1)=(0,-1)$. Moreover, if $\operatorname{pr} \circ \iota_{\xi, \xi^{\prime}}(p)=(a, b)$ with $a \neq 0$, then $\operatorname{pr} \circ \iota_{\xi, \xi^{\prime}}(q)=\left(\frac{b^{2}-1}{a}, b\right)$.

3.3.1. Remark. We also need an analogue of Lemma 3.3 in the target space, i.e., for $\mathfrak{C}_{g}$. This can be seen by a direct computation which involves solving a quadratic equation. As was done in the proof of Lemma 3.3. one may also simplify this computation as follows: We may reduce to the case where the graph is given by $z w=1, \xi=(\infty, \infty), \xi^{\prime}=(r, s)$ with $r, s$ both finite and every line $a z+b$ through $\xi^{\prime}$ intersects $z w=1$. Then, on the finite points of the graph, the inversion is given by $z \mapsto-z-\frac{b}{a}$.

3.4. Lemma. Let $E^{\prime} \subset E_{C}$ be a set with positive measure. Then the group generated by the set of inversions induced by $\left(\xi, \xi^{\prime}\right) \in E^{\prime}$, see Lemma 3.3, contains $\mathrm{PSL}_{2}(\mathbb{R})$.

Proof. Let $\mathcal{I}^{\prime}$ be the set of inversions induced by $E^{\prime}$. Then

$$
\mathcal{I}^{\prime} \subset \operatorname{Inv}:=\left\{\left(\begin{array}{cc}
a & b \\
c & -a
\end{array}\right): a, b, c \in \mathbb{R}, a^{2}+b c=-1\right\},
$$

and if we equip Inv with the Lebesgue measure, $\mathcal{I}^{\prime}$ has positive measure. Any set of positive measure in Inv generates $\mathrm{PSL}_{2}(\mathbb{R})$. 
To see this, note that for a.e. $\left(g, g^{\prime}\right) \in \operatorname{Inv}$ we have $g \operatorname{Inv} \cap g^{\prime} \operatorname{Inv}$ is one dimensional. Therefore, there are $g, g^{\prime} \in \mathcal{I}^{\prime}$ so that $Q:=\left(g \mathcal{I}^{\prime}\right)\left(g^{\prime} \mathcal{I}^{\prime}\right)$ has positive measure in $\operatorname{PSL}_{2}(\mathbb{R})$. Now $Q^{-1} Q$ contains an open neighborhood of the identity and $\mathrm{PSL}_{2}(\mathbb{R})$ is connected, hence, $Q^{-1} Q$ generates a subgroup which contains $\mathrm{PSL}_{2}(\mathbb{R})$.

Let the notation be as in Proposition 3.1. In particular,

$$
\Psi: \mathbb{S}^{2} \rightarrow \mathbb{P l}_{v} \times \mathbb{P l}_{v}
$$

is a $\Gamma$-equivariant measurable map in Proposition 3.1

3.5. Lemma. Let $\mathcal{V}=\left\{\left(C, \theta, \theta^{\prime}\right) \in \mathcal{C} \times \mathbb{S}^{2} \times \mathbb{S}^{2}: \theta, \theta^{\prime} \in C\right\}$. Then $\mathcal{V}$ is a subvariety of $\mathcal{C} \times \mathbb{S}^{2} \times \mathbb{S}^{2}$; equip $\mathcal{V}$ with the natural measure. There exists a conull Borel measurable subset

$$
\mathcal{A} \subset \mathcal{V}
$$

so that $\Psi(\theta), \Psi\left(\theta^{\prime}\right)$ belong to the essential image of $\left.\Psi\right|_{C}$ for all $\left(C, \theta, \theta^{\prime}\right) \in \mathcal{A}$, where $\Psi$ is as in Proposition 3.1 .

Proof. Since $\Psi$ is measurable, for any $n \in \mathbb{N}$ there exists a compact subset $D_{n} \subset \mathbb{S}^{2}$ with $\mathrm{m}\left(\mathbb{S}^{2} \backslash D_{n}\right)<1 / n$ so that $\left.\Psi\right|_{D_{n}}$ is continuous; we may also assume that $D_{1} \subset D_{2} \subset \cdots$.

For every $m \in \mathbb{N}$, let $\mathcal{C}_{m}$ denote the set of circles with radius $\geq 1 / m$. Then $\mathcal{C}_{m}$ is a compact subset of $\mathcal{C}$ and $\mathcal{C}=\cup \mathcal{C}_{m}$. For every $m, n \in \mathbb{N}$ let $\mathcal{C}_{m, n}=\left\{C \in \mathcal{C}_{m}: \mathrm{m}_{C}\left(C \cap D_{n}\right)>0\right\}$. Then $\cup_{n} \mathcal{C}_{m, n}$ is a conull Borel subset of $\mathcal{C}_{m}$ for every $m$.

Let $C \in \mathcal{C}_{m, n}$ and let $\theta \in C \cap D_{n}$ be a density point for $C \cap D_{n}$. Then by continuity of $\left.\Psi\right|_{D_{n}}$, for every $r>0$, we have $\Psi^{-1}\left(\mathrm{~N}_{r}(\Psi(\theta)) \cap D_{n} \cap C\right.$ is an open subset of $D_{n} \cap C$; since $\theta$ is a density point of $C \cap D_{n}$, we get that $\mathrm{m}_{C}\left(\Psi^{-1}\left(\mathrm{~N}_{r}(\Psi(\theta)) \cap D_{n} \cap C\right)>0\right.$. In particular, $\Psi(\theta)$ belongs to the essential image of $\left.\Psi\right|_{C}$.

Define $\mathcal{A}_{m, n}^{\prime}=\left\{\left(C, \theta, \theta^{\prime}\right): C \in \mathcal{C}_{m, n}, \theta, \theta^{\prime} \in C \cap D_{n}\right\}$. Then $\mathcal{A}_{m, n}^{\prime}$ is a Borel subset of $\mathcal{V}$. Using a countable basis of open subsets, we see that

$$
\mathcal{A}_{m, n}=\left\{\left(C, \theta, \theta^{\prime}\right) \in \mathcal{A}_{m, n}^{\prime}: \theta, \theta^{\prime} \text { are density points of } C \cap D_{n}\right\}
$$

is a conull subset of $\mathcal{A}_{m, n}^{\prime}$.

This in view of the above argument implies that $\mathcal{A}=\cup_{m, n} \mathcal{A}_{m, n}$ satisfies the claim in the lemma.

3.6. Lemma. Let the notation be as in Proposition 3.1. For a.e. $C \in \mathcal{C}$, there is a conull subset $E_{C}^{\prime} \subset E_{C}$, see (3.2), so that for all $\left(\xi, \xi^{\prime}\right) \in E_{C}^{\prime}$ the following hold.

(1) The essential image of $\left.\Psi\right|_{C}$ is a subset of $\mathfrak{C}_{g_{C}}$ and is an infinite subset.

(2) $\Psi(\xi) \neq \Psi\left(\xi^{\prime}\right)$.

(3) For a.e. $t \in I_{\xi, \xi^{\prime}}$, there exists $\mathfrak{C}_{t}$ so that the essential image of $\left.\Psi\right|_{C_{t}\left(\xi, \xi^{\prime}\right)}$ contained in $\mathfrak{C}_{t}$. 
(4) Let $C \cap C_{t}\left(\xi, \xi^{\prime}\right)=\left\{\theta, \theta^{\prime}\right\}$. Then $\Psi(\theta) \neq \Psi\left(\theta^{\prime}\right)$, moreover, they both belong to the essential image of $\left.\Psi\right|_{C}$.

Proof. Let $\mathcal{C}^{\prime} \subset \mathcal{C}$ be a conull subset where Proposition 3.1(1) and (2) hold. Then, for any $C \in \mathcal{C}^{\prime}$ part (1) in this lemma holds.

Applying Lemma 2.7(1) with $\Psi$ and $\Psi(\xi)$ (for every $\xi \in \mathbb{S}^{2}$ so that $\Psi(\xi)$ is defined), the set of $\xi^{\prime} \in \mathbb{S}^{2}$ such that $\Psi\left(\xi^{\prime}\right)=\Psi(\xi)$ is a null set. That is: for a.e. $\left(\xi, \xi^{\prime}\right) \in \mathbb{S}^{2} \times \mathbb{S}^{2}$ we have $\Psi(\xi) \neq \Psi\left(\xi^{\prime}\right)$.

Note also that since $\mathcal{C}^{\prime}$ is conull, for a.e. $\left(\xi, \xi^{\prime}\right) \in \mathbb{S}^{2} \times \mathbb{S}^{2}$ we have

$$
C_{t}\left(\xi, \xi^{\prime}\right) \in \mathcal{C}^{\prime} \quad \text { for a.e. } t \in I_{\xi, \xi^{\prime}} \text {. }
$$

In consequence, for a.e. $\left(\xi, \xi^{\prime}\right) \in E_{C}$ parts (2) and (3) hold true. We now show that (4) also holds for a.e. $C \in \mathcal{C}^{\prime}$ and a.e. $\left(\xi, \xi^{\prime}\right) \in E_{C}$.

Define $\mathcal{A}^{\prime}:=\mathcal{A} \cap \operatorname{pr}^{-1}\left(\mathcal{C}^{\prime}\right)$ where pr $: \mathcal{V} \rightarrow \mathcal{C}$ is the projection map and $\mathcal{A}$ is as in Lemma 3.5. Let $\mathcal{B}^{\prime} \subset \mathcal{A}^{\prime} \times \mathbb{S}^{2} \times \mathbb{S}^{2} \times \mathbb{R}$ be the set of points $\left(C, \theta, \theta^{\prime}, \xi, \xi^{\prime}, t\right)$ where $\left(C, \theta, \theta^{\prime}\right) \in \mathcal{A}^{\prime},\left(\xi, \xi^{\prime}\right) \in E_{C}$, and $t \in I_{\xi, \xi^{\prime}}$ - then $\mathcal{B}^{\prime}$ is Borel subset. Let

$$
\mathcal{B}:=\left\{\left(C, \theta, \theta^{\prime}, \xi, \xi^{\prime}, t\right) \in \mathcal{B}^{\prime}: C \cap C_{t}\left(\xi, \xi^{\prime}\right)=\left\{\theta, \theta^{\prime}\right\}\right\} ;
$$

note that $\mathcal{B}$ is also a Borel set. Indeed, $\mathcal{B}$ is the inverse image of the diagonal in $\left(\left(\mathbb{S}^{1} \times \mathbb{S}^{1}\right) /(\mathbb{Z} / 2)\right) \times\left(\left(\mathbb{S}^{1} \times \mathbb{S}^{1}\right) /(\mathbb{Z} / 2)\right)$ under the map $\left(C, \theta, \theta^{\prime}, \xi, \xi^{\prime}, t\right) \mapsto$ $\left(\left\{\theta, \theta^{\prime}\right\}, C \cap C_{t}\left(\xi, \xi^{\prime}\right)\right)$ where we identified $C$ with $\mathbb{S}^{1}$.

By the definition of $\mathcal{B}$ and Lemma 3.5. for any $\left(C, \theta, \theta^{\prime}, \xi, \xi^{\prime}, t\right) \in \mathcal{B}$ we have $\Psi(\theta)$ and $\Psi\left(\theta^{\prime}\right)$ belong to the essential image of $\left.\Psi\right|_{C}$.

Observe that if we fix $(C, \theta)$ and vary $\left(\xi, \xi^{\prime}\right) \in E_{C}$ and $t \in I_{\xi, \xi^{\prime}}$, we cover every $\theta \neq \theta^{\prime} \in C$. It thus follows, from the implicit function theorem, that for every $C$, a.e. $\left(\xi, \xi^{\prime}\right) \in E_{C}$, and a.e. $t \in I_{\xi, \xi^{\prime}}$, there exists some $\left(\theta, \theta^{\prime}\right)$ so that $\left(C, \theta, \theta^{\prime}, \xi, \xi^{\prime}, t\right) \in \mathcal{B}$ satisfies the second claim in part (4) holds.

We now show the first claim in (4) also holds, possibly after removing another null subset. Let $\check{\mathcal{B}} \subset \mathcal{B}$ be the set of $\left(C, \theta, \theta^{\prime}, \xi, \xi^{\prime}, t\right) \in \mathcal{B}$ so that $\Psi(\theta)=\Psi\left(\theta^{\prime}\right)$. We claim that $\check{\mathcal{B}}$ is a null set; this finishes the proof of (4) and the lemma.

Assume to the contrary that $\check{\mathcal{B}}$ has positive measure. By Fubini's theorem, thus, there exists some $(C, \theta)$ so that

$$
\check{\mathcal{B}}_{(C, \theta)}:=\left\{\left(\theta^{\prime}, \xi, \xi^{\prime}, t\right):\left(C, \theta, \theta^{\prime}, \xi, \xi^{\prime}, t\right) \in \check{\mathcal{B}}\right\}
$$

has positive measure.

Using the implicit function theorem, we get a subset $J \subset C$ with $\mathrm{m}_{C}(J)>$ 0 so that $\Psi\left(\theta^{\prime}\right)=\Psi(\theta)$ for all $\theta^{\prime} \in J$. This contradicts the fact that $C \in \mathcal{C}^{\prime}$ - recall that Proposition 3.1(1) holds for all $C \in \mathcal{C}^{\prime}$.

3.7. Lemma. Let the notation be as in Proposition 3.1. In particular,

$$
\Psi: \mathbb{S}^{2} \rightarrow \mathbb{P l}_{v} \times \mathbb{P l}_{v}
$$

is a $\Gamma$-equivariant measurable map which satisfies parts (1) and (2) in Proposition 3.1. Then $\mathfrak{l}_{v}=\mathbb{C}$ and $\Psi$ agrees with a rational map from $\mathbb{S}^{2}$ into $\mathbb{P C} \times \mathbb{P C}$ almost everywhere. 
Proof. Let $C \in \mathcal{C}$ be so that Lemma 3.6 holds true and let $E_{C}^{\prime} \subset E_{C}$ be as in loc. cit. In particular the essential image of $\left.\Psi\right|_{C}$ belongs to $\mathfrak{C}_{g_{C}}$. Let $\mathcal{I}$ be the collection of inversions of $C$ obtained by $\left(\xi, \xi^{\prime}\right) \in E_{C}^{\prime}$, see Lemma 3.3. Then by Lemma 3.4, $\mathcal{I}$ generates $\mathrm{PSL}_{2}(\mathbb{R})$.

Recall that the essential image of $\left.\Psi\right|_{C}$ in $\mathfrak{C}$ is infinite, therefore, an inversion is uniquely determined by its restriction to the essential image of $\Psi$. By Lemma 3.6 and Remark 3.3.1, $\Psi$ induces a map $f$ from $\mathcal{I}$ into the set of inversions on $\mathfrak{C}$.

Since the essential image of $\left.\Psi\right|_{C}$ in $\mathfrak{C}$ is infinite, we get from Lemma 3.2 that $f$ extends to a continuous homomorphism from $\mathrm{PSL}_{2}(\mathbb{R})$ into $\mathrm{PGL}_{2}\left(\mathfrak{l}_{v}\right)$. Such a homomorphism can only arise from algebraic constructions as follows: There exists a continuous homomorphism of fields $\vartheta: \mathbb{R} \rightarrow \mathfrak{l}_{v}$ and an isomorphism of algebraic groups $\varphi:{ }^{\vartheta} \mathrm{PGL}_{2} \rightarrow \mathrm{PGL}_{2}$ so that $f(g)=\varphi\left(\vartheta^{0}(g)\right)$ for all $g \in \mathrm{PSL}_{2}(\mathbb{R})$ where $\vartheta^{0}: \mathrm{PGL}_{2}(\mathbb{R}) \rightarrow \mathrm{PGL}_{2}(\vartheta(\mathbb{R}))$ is the isomorphism induced by $\vartheta$, see [22, Ch. I, $\S 1.8]$.

Since there are no monomorphism from $\mathbb{R}$ into non-Archimedean local fields, continuous or not, we get that $\mathfrak{l}_{v}=\mathbb{C}$.

We now show that $\Psi$ agrees with a rational map ${ }^{1}$ almost surely. Let $C$ and $C^{\prime}$ be two circles which intersect at two points and both satisfy Lemma 3.6 - the set of intersecting circles has positive measure in $\mathcal{C}$, hence, two such circles exist. Let $C \cap C^{\prime}=\left\{\xi, \xi^{\prime}\right\}$ where $\xi \neq \xi^{\prime}$. Using the stereographic projection of $\mathbb{S}^{2}$ with $\xi$ as the pole, we get coordinates on $\mathbb{R}^{2}$ induced by two lines $\ell$ and $\ell^{\prime}$ corresponding $C$ and $C^{\prime}$, respectively, which intersect at the image of $\xi^{\prime}$. Thus $\Psi$ induces a measurable map in two variables which is rational in each variable. In view of [21, Lemma 17], we thus get that $\Psi$ agrees with a rational map almost surely.

3.8. Proofs of the main theorems. In this section we complete the proofs of Theorem 1.2 and Theorem 1.1 assuming Proposition 3.1 .

Proof of Theorem 1.2. In view of Lemma 3.7 we may assume $\mathfrak{l}_{v}=\mathbb{C}$ and $\Psi$ agrees with a rational map almost surely. Theorem 1.2 follows from this by [21, §1.3] as the action of $\mathrm{PGL}_{2} \times \mathrm{PGL}_{2}$ on its boundary is strictly effective.

Proof of Theorem 1.1. We recall the argument from [21, Proof of Thm. 1, p. 97]. Let the notation be as in $\$ 2.1$; in particular, $v_{0}$ and $\sigma=\mathrm{id}$ are the place and the embedding which give rise to the lattice $\Gamma$ in $\mathrm{PGL}_{2}(\mathbb{C})$. By Theorem 1.2 for any $(v, \sigma) \neq\left(v_{0}\right.$,id) we have $\sigma(\Gamma)$ is bounded in $\mathbf{G}\left(\mathrm{L}_{v}\right)$.

Let $\mathbf{G}^{\prime}=\mathrm{R}_{\mathrm{L} / \mathbb{Q}}(\mathbf{G})$ where $\mathrm{R}_{\mathrm{L} / \mathbb{Q}}$ is the restriction of scalars. Then $\mathbf{G}^{\prime}(\mathbb{R})$ is naturally identified with $\prod \mathbf{G}\left(\mathrm{L}_{v}\right)$ where the product is taken over all the Archimedean places. Let $\varphi(\Gamma)$ denote the image of $\Gamma$ in $\mathbf{G}^{\prime}$ - note that $\varphi(\Gamma)$ is isomorphic to $\Gamma$ and $\varphi(\Gamma) \subset \mathbf{G}^{\prime}(\mathbb{Q})$.

\footnotetext{
${ }^{1}$ In the case at hand this assertion can be proved using more elementary arguments, e.g. by choosing three parallel circles.
} 
Let $\mathbf{F}$ be the Zariski closure of $\varphi(\Gamma)$ in $\mathbf{G}^{\prime}$. Then the natural map $\varrho$ : $\mathbf{F} \rightarrow \mathbf{G}$ is an $\mathbb{R}$-epimorphism. Let $\mathbf{K}=\operatorname{ker}(\varrho)$. Recall that any compact subgroup of a real algebraic group is itself algebraic. In view of this and since $\varphi(\Gamma)$ has bounded image in $\mathbf{K}(\mathbb{R})$, we get that $\mathbf{K}(\mathbb{R})$ is compact.

Moreover, $\sigma(\Gamma)$ is bounded in $\mathbf{G}\left(\mathrm{L}_{v}\right)$ for all non-Archimedean places and $\Gamma$ is finitely generated, hence, $\varphi(\Gamma) \cap \mathbf{F}(\mathbb{Z})$ in finite index in $\varphi(\Gamma)$. Further, since $\mathbf{K}(\mathbb{R})$ is compact, $\varrho(\mathbf{F}(\mathbb{Z}))$ is discrete in $\mathbf{G}(\mathbb{R})$; we get that $\Gamma$ and $\varrho(\mathbf{F}(\mathbb{Z}))$ are commensurable. The proof is complete.

\section{The COCYCle AND EQUivariant MEASURABle MAPS}

In this section we recall a construction due to Margulis which produces an equivariant measurable map between certain projective spaces.

Let $\Gamma \subset G$ be a uniform lattice and let $\mathfrak{l}$ be a local field of characteristic zero. We assume fixed a homomorphism

$$
\rho: \Gamma \rightarrow \mathrm{PGL}_{2}(\mathfrak{l})
$$

whose image is unbounded and Zariski dense - in our application, $\rho$ will actually be a monomorphism.

4.1. Characteristic maps and cocylces. Let $B$ denote the group of upper triangular matrices in $\mathrm{PGL}_{2}(\mathfrak{l})$. We now recall from [22, $\mathrm{Ch}$. V] the construction of a measurable map from $Q \backslash G$ to $B \backslash \mathrm{PGL}_{2}(\mathfrak{l})$ which is associated to the representation $\rho$. This approach relies on the multiplicative ergodic theorem.

We fix a fundamental domain $F$ which is the invariant lift of a Dirichlet fundmental domain for $\Gamma$ in $\mathbb{H}^{3}$ - in particular, $F$ is compact and $\partial F$ is a union of finitely many suborbiforlds of lower dimension.

For any $g \in G$, there exists a unique $\gamma_{g} \in \Gamma$ so that $g \in F \gamma_{g}$. Set

$$
\omega(g):=\rho\left(\gamma_{g}\right) .
$$

Then $\omega: G \rightarrow \mathrm{PGL}_{2}(\mathfrak{l})$ is a Borel map and

$$
\omega(g \gamma)=\omega(g) \rho(\gamma) \text { for all } g \in G \text { and } \gamma \in \Gamma .
$$

Define $b_{\omega}^{\prime}(g, y)=\omega(y) \omega(g y)^{-1}$ for all $g \in G$ and $y \in G$. Note that by (4.1) we have $b_{\omega}^{\prime}(g, y)=b_{\omega}^{\prime}(g, y \gamma)$ for all $\gamma \in \Gamma$. Define

$$
b_{\omega}(g, x)=b_{\omega}^{\prime}\left(g, \pi^{-1}(x)\right) \text { for all } g \in G \text { and } x \in X \text {. }
$$

Then $b_{\omega}\left(g_{1} g_{2}, x\right)=b_{\omega}\left(g_{2}, x\right) b_{\omega}\left(g_{1}, g_{2} x\right)$. That is: $b_{\omega}$ is a cocycle.

Define the cocycle

$$
u(n, x)=b_{\omega}\left(a_{n}, x\right) \text { for all } x \in X \text { and all } n \in \mathbb{Z} ;
$$

where $a_{t}$ is defined in (2.1).

4.2. Theorem (Cf. 22], Ch. V and VI). Let the notation be as above.

(1) There exists a unique $\Gamma$-equivariant Borel measurable map

$$
\psi: \mathbb{S}^{2} \rightarrow B \backslash \mathrm{PGL}_{2}(\mathfrak{l}) .
$$


(2) There exist some $\lambda_{1}>0$ so that the following holds. Let $w \in \mathfrak{l}^{2} \backslash\{0\}$. Then for $\operatorname{vol}_{X}$-a.e. $x \in X$ we have

$$
\lim _{n \rightarrow \infty} \frac{1}{n} \log (\|u(n, x) w\| /\|w\|)=\lambda_{1}
$$

Proof. The existence of a measurable map $\psi$ as in (1) is proved in 22 , Ch. V, Thm. 3.2]. An alternative approach is [22, Ch. VI, Thm. 4.3] which is based on the work of Furstenberg.

The fact that there exists $\lambda_{1}>0$ so that part (2) holds follows from the fact that $\rho(\Gamma)$ is unbounded and Zariski dense, see [12, 14] and [22, Ch. V, Thm. 3.2].

4.3. A set of uniform convergence. Let $\varepsilon>0$. Recall that $F$ is the invariant lift of a compact Dirichlet fundamental domain $E$ for the action of $\Gamma$ on $\mathbb{H}^{3}$. Let $\mathcal{C}_{E, \varepsilon} \subset F$ be as in Lemma 2.8.

Let $e_{1}=(1,0) \in \mathfrak{l}^{2}$. There exists a compact subset $F_{\varepsilon}^{\prime} \subset \mathcal{C}_{E, \varepsilon}$ with $\operatorname{vol}_{X}\left(F_{\varepsilon}^{\prime}\right)>1-\varepsilon^{8}$ so that the convergence in Theorem 4.2(2) is uniform. That is for every $\eta>0$ there exists some $n_{\eta}$ so that for all $n>n_{\eta}$ and any $x \in F_{\varepsilon}^{\prime}$ we have

$$
\left|\left(\frac{1}{n} \log \left\|u(n, x) e_{1}\right\|\right)-\lambda_{1}\right|<\eta \quad \text { for all } n>n_{\eta} .
$$

Since the measure $\operatorname{vol}_{X}$ is $G$-invariant and in particular, $\mathrm{SO}(2)$-invariant, the following holds. There exists a compact subset $F_{\varepsilon} \subset F_{\varepsilon}^{\prime}$ with

$$
\operatorname{vol}_{X}\left(F_{\varepsilon}\right)>1-\varepsilon^{4}
$$

so that for every $g \in F_{\varepsilon}$ we have $\left|\left\{\theta \in[0,2 \pi]: r_{\theta} g \in F_{\varepsilon}^{\prime}\right\}\right|>1-\varepsilon^{4}$ where $r_{\theta}$ denotes the rotation matrix with angle $\theta$.

Let $\tau>0$ be fixed. For every $\alpha>0$, let $F_{\varepsilon}(\tau, \alpha) \subset F_{\varepsilon}$ be the subset with the property that for every $g \in F_{\varepsilon}(\tau, \alpha)$ we have

$$
\left|\left\{\theta \in[0,2 \pi]: \mathrm{B}\left(a_{\tau} r_{\theta} g, \alpha\right) \subset F \omega\left(a_{\tau} r_{\theta} g\right)\right\}\right|>2\left(1-2 \varepsilon^{4}\right) 2 \pi
$$

where for every $h \in G$ we have $\omega(h) \in \Gamma$ is the element so that $h \in F \omega(h)$ and $\mathrm{B}(h, \alpha)$ denote the ball of radius $\alpha$ centered at $h$.

Let $\tau$ and $\varepsilon>0$ be fixed. There exists some $\alpha_{0}=\alpha_{0}(\varepsilon)$ so that

$$
\operatorname{vol}\left(F_{\varepsilon}\left(\tau, \alpha_{0}\right)\right)>1-2 \varepsilon^{4} .
$$

Random walks and distances. Let $\mathcal{T}$ denote the Bruhat-Tits tree (if $\mathfrak{l}$ is non-Archimedean) or $\mathbb{H}^{3}$ if $\mathfrak{l}=\mathbb{C}$. Let $\mathrm{d}_{\mathcal{T}}$ denote the $\mathrm{PGL}_{2}(\mathfrak{l})$-invariant metric on $\mathcal{T}$ - that is: if $\mathfrak{l}=\mathbb{C}$, then $\mathrm{d}_{\mathcal{T}}$ is the hyperbolic metric and if $\mathfrak{l}$ is non-Archimedean, then $\mathrm{d}_{\mathcal{T}}$ is the path metric on a tree. We fix a base point $o \in \mathcal{T}$ which we assume to be the image of the identity element of $\mathrm{PGL}_{2}(\mathfrak{l})$ in $\mathcal{T}$. Abusing the notation, given an element $g \in \mathrm{PGL}_{2}(\mathfrak{l})$ we often write $\mathrm{d}_{\mathcal{T}}(g, o)$ for $\mathrm{d}_{\mathcal{T}}(g \cdot o, o)$.

Recall that $\Gamma$ is a uniform lattice in $G$. Therefore,

$$
\mathrm{d}_{\mathcal{T}}(u(n, x), o) \leq L_{1} n
$$


for some $L_{1}$ depending on the representation $\rho$.

4.4. Lemma. Let $0<\varepsilon<1 / 2$ be so that $\left[L_{1}=<0.01 \lambda_{1}\right.$. Let $F_{\varepsilon}$ be defined as in 4.3 . There exists some $N_{0}$ so that for any $n>N_{0}$ we have the following.

(1) For every geodesic $\xi=\left\{\xi_{t}\right\} \subset \mathcal{T}$ with $\xi_{0}=o$ and every $g \in F_{\varepsilon}$, there exists a subset $R_{g, \xi} \subset[0,2 \pi]$ with $\left|R_{g, \xi}\right|>2(1-\varepsilon) \pi$ so that for all $\theta \in R_{g, \xi}$ we have

$$
\mathrm{d}_{\mathcal{T}}\left(u\left(n, r_{\theta} g\right) \cdot o, \xi_{t}\right)>t+\lambda_{1} n / 3 .
$$

(2) For every geodesic $\xi=\left\{\xi_{t}\right\} \subset \mathcal{T}$ with $\xi_{0}=o$ and every $g \in F_{\varepsilon}$ we have

$$
\frac{1}{2 \pi} \int_{0}^{2 \pi} \mathrm{d} \mathcal{T}\left(u\left(n, r_{\theta} g\right) \cdot o, \xi\right) \mathrm{d} \theta>t+\lambda_{1} n / 5
$$

Proof. The proof is a special case of the argument presented in the proof of Lemma 2.5. we repeat parts of the argument for the convenience of the reader. sion.

Apply (4.3) with $\eta=1 / 2$ and assume $n>n_{1 / 2}$ for the rest of the discus-

For any $\theta \in[0,2 \pi]$, let $q_{\theta}=u\left(n, r_{\theta} g\right) \cdot o$. By the hyperbolic law of cosines, see (2.3), we have

$$
\cosh \left(\overline{q_{\theta} \xi_{t}}\right)=\cosh \left(\overline{o \xi_{t}}\right) \cosh \left(\overline{o q_{\theta}}\right)-\cos (\alpha) \sinh \left(\overline{o \xi_{t}}\right) \sinh \left(\overline{o q_{\theta}}\right)
$$

where $\alpha$ is the angle between $\overline{o \xi_{t}}$ and $\overline{o q_{\theta}}$.

Then in view of Lemma 2.8 and the fact that $g \in F_{\varepsilon} \subset \mathcal{C}_{E, \varepsilon}$, there exists some $\delta>0$ so that

$$
\left|\left\{\theta \in[0,2 \pi]: \psi\left(r_{\theta} g\right) \notin \mathrm{N}_{\delta}\left(\xi_{\infty}\right)\right\}\right|>2(1-\varepsilon) \pi
$$

where $\xi_{\infty}=\lim _{t \rightarrow \infty} \xi_{t} \in \mathrm{PGL}_{2}(\mathfrak{l}) / B$.

Therefore, we have

$$
\mathrm{d}_{\mathcal{T}}\left(u\left(n, r_{\theta} g\right) \cdot o, \xi_{t}\right)>t+\frac{\lambda_{1} n}{2}-O_{\delta}(1)
$$

for all $\theta$ which lies in the set appearing in (4.7). Assuming $n$ is large enough, depending on $\delta$, we get part (1).

Recall that $\mathrm{d}_{\mathcal{T}}(u(n, h) \cdot o, o) \leq L_{1}$ for all $h \in F$. In particular we have

$$
\mathrm{d}_{\mathcal{T}}\left(u(n, h) \cdot o, \xi_{t}\right) \geq t-L_{1}^{n} \quad \text { for all } n .
$$

Part (2) now follows from (4.8) and (4.9) as Lemma 2.5 was proved using (2.8) and (2.9).

4.5. Lemma. Let $0<\varepsilon<1 / 2$ with $L_{1}=0.01 \lambda_{1}$ and let $\tau>N_{0}$ be a fixed parameter, where $N_{0}$ is as in Lemma 4.4. Suppose $x \in X$ is so that there exists some $g_{x} \in F_{\varepsilon}\left(\tau, \alpha_{0}\right)$ with

$$
\operatorname{dist}\left(a_{\tau} r_{\theta} g_{x}, a_{\tau} r_{\theta} x\right)<\alpha_{0} / 2 \text { for all } \theta \in[0,2 \pi] \text {, }
$$

see (4.5). Then the following hold. 
(1) Let $\xi=\left\{\xi_{t}\right\} \subset \mathcal{T}$ with $\xi_{0}=o$ be a geodesic. There exists a subset $\hat{R}_{x, \xi} \subset[0,2 \pi]$ with $\left|\hat{R}_{x, \xi}\right|>2(1-2 \varepsilon) \pi$ so that for all $\theta \in \hat{R}_{x, \xi}$ we have

$$
\mathrm{d}_{\mathcal{T}}\left(u\left(\tau, r_{\theta} x\right), \xi_{t}\right)>t+\tau \lambda_{1} / 3
$$

(2) Let $\xi=\left\{\xi_{t}\right\} \subset \mathcal{T}$ with $\xi_{0}=o$ be a geodesic. Then

$$
\frac{1}{2 \pi} \int_{0}^{2 \pi} \mathrm{d}_{\mathcal{T}}\left(u\left(\tau, r_{\theta} x\right), \xi_{t}\right) \mathrm{d} \theta>t+\tau \lambda_{1} / 5
$$

Proof. We first prove part (1). Let

$$
R_{g_{x}}=\left\{\theta \in[0,2 \pi]: \mathrm{B}\left(a_{\tau} r_{\theta} g_{x}, \alpha_{0}\right) \subset F\right\} .
$$

Then by the definition of $F_{\varepsilon}\left(\tau, \alpha_{0}\right)$, see (4.4), we have $\left|R_{g_{x}}\right|>2\left(1-2 \varepsilon^{4}\right) \pi$.

Let $R_{g_{x}, \xi}$ be as in Lemma 4.4(1) applied to $g_{x}$ and $\xi$ and put

$$
\hat{R}_{x, \xi}:=R_{g_{x}} \cap R_{g_{x}, \xi}
$$

Note that $\left|\hat{R}_{x, \xi}\right|>2(1-2 \varepsilon) \pi$.

Let now $\theta \in \hat{R}_{x, k}$. By (4.10), we have

$$
\operatorname{dist}\left(a_{\tau} r_{\theta} g_{x}, a_{\tau} r_{\theta} x\right)<\alpha_{0} / 2 \text {. }
$$

Since $\theta \in R_{x, g_{x}}$, we have $u\left(\tau, r_{\theta} x\right)=u\left(\tau, r_{\theta} g_{x}\right)$. Moreover, since $\theta \in R_{g_{x}, \xi}$ and $\tau>N_{0}$, we get from Lemma 4.4)(1) that

$$
\mathrm{d}_{\mathcal{T}}\left(u\left(\tau, r_{\theta} x\right), \xi_{t}\right)=\mathrm{d}_{\mathcal{T}}\left(u\left(\tau, r_{\theta} g_{x}\right), \xi_{t}\right)>t+\lambda_{1} \tau / 3,
$$

as was claimed in part (1).

The proof of part (2) is similar to the proof Lemma 2.5 as we now explicate. Recall from (4.6) that

$$
\mathrm{d}_{\mathcal{T}}\left(u\left(\tau, r_{\theta} x\right), \xi_{t}\right) \geq t-L_{1} \tau
$$

for any $\theta \in[0,2 \pi]$.

Using part (1) and (4.11) we obtain the following.

$$
\begin{aligned}
\frac{1}{2 \pi} \int_{0}^{2 \pi} \mathrm{d}_{\mathcal{T}}\left(u\left(\tau, r_{\theta} x\right), \xi_{t}\right) \mathrm{d} \theta & >(1-2 \varepsilon)\left(t+\frac{\lambda_{1} \tau}{3}\right)+2\left(t-L_{1} \tau\right) \varepsilon \\
& >t+\lambda_{1} \tau / 4-2 L_{1} \lambda_{1} \varepsilon \tau \\
L_{1}<0.01 \lambda_{1} \rightsquigarrow & >t+\lambda_{1} \tau / 5 .
\end{aligned}
$$

The proof if complete.

\section{The MAIN LEMMA}

The following lemma is one of the pivotal ingredients in the proof of Proposition 3.1, and is one of the main technical tools in this paper.

5.1. Lemma (Main Lemma). Let the notation be as in 94 Further, assume that there are infinitely many closed $H$-orbits $\left\{H x_{i}: i \in \mathbb{N}\right\}$ in $X=G / \Gamma$. There exists some $\lambda_{0}=\lambda_{0}(\rho)>0$ with the following property. 
For every $\varepsilon>0$, there exist positive integers $i_{0}, \tau$, and $N$ with the following properties. For all $i>i_{0}$, there exists a subset $Z_{i} \subset H x_{i}$ with $\mu_{H x_{i}}\left(Z_{i}\right)>1-\varepsilon$ so that

$$
\mathrm{d}_{\mathcal{T}}(u(n \tau, z), o)>\lambda_{0} \tau n \quad \text { for all } z \in Z_{i} \text { and all } n>N .
$$

The proof of this lemma relies on results in $\$ 4$, equidistribution theorems in homogeneous dynamics, and certain maximal inequalities. The proof will occupy the rest of this section.

We begin with the following theorem which is a special case of a theorem of Mozes and Shah [24 - the proof in 24 builds on seminal works on unipotent dynamics by Dani, Margulis, and Ratner.

5.2. Theorem. Let $\Gamma \subset G$ be a lattice and let $\operatorname{vol}_{X}$ denote the probability $G$-invariant measure on $X=G / \Gamma$. Assume that there are infinitely many closed $H$-orbits $\left\{H x_{i}: i \in \mathbb{N}\right\}$ in $X$. For every $i$ let $\mu_{H x_{i}}$ denote the $H$ invariant probability measure on $H x_{i}$. Then

$$
\int f \mathrm{~d} \mu_{H x_{i}} \rightarrow \int f \mathrm{dvol} \text { for any } f \in C_{c}(G / \Gamma) .
$$

This theorem plays an important role in the sequel. We record a corollary of this theorem here which will be used in 96 .

5.2.1. Corollary. Let the notation be as in Theorem 5.2. Let $0<\varepsilon<1 / 2$ and for each $i$, let $Z_{i} \subset H x_{i}$ be a subset with $\mu_{H x_{i}}\left(Z_{i}\right)>1-\varepsilon$. Let $\delta>0$ and for each $i$, let $\mathcal{N}_{i, \delta}$ be the open $\delta$-neighborhood of $Z_{i}$. Then there exists some $i_{1}$ so that

$$
\operatorname{vol}_{X}\left(\mathcal{N}_{i, \delta}\right)>1-3 \varepsilon \text { for all } i>i_{1} .
$$

Proof. For each $i$ consider a covering of $X \backslash \mathcal{N}_{i, \delta}$ with balls of radius $\delta / 2$ with multiplicity $\kappa$ depending only on $X$. The characteristic function of these open sets can be approximated by a precompact family $\mathcal{F}$ of continuous functions. The claim follows since Theorem 5.2 holds uniformly on precompact families.

5.3. Maximal inequalities. Let $Y=H x \subset X$ be a closed $H$-orbit and let $\mu$ be the probability $H$-invariant measure on $Y$. Let $\mathcal{R}=[0,2 \pi]^{\mathbb{Z}}$ be equipped with $\mathrm{d} \nu:=\left(\frac{\mathrm{d} \theta}{2 \pi}\right)^{\otimes \mathbb{Z}}$.

Let $\tau>0$ and define $\eta_{Y}: \mathcal{R} \times Y \rightarrow \mathcal{R} \times Y$ by

$$
\eta_{Y}\left(\left(\theta_{n}\right), y\right)=\left(\eta\left(\theta_{n}\right), a_{\tau} r_{\theta_{1}} y\right)
$$

where $\eta: \mathcal{R} \rightarrow \mathcal{R}$ is the shift map. Then the measure $\nu \times \mu$ is $\eta_{Y}$-invariant and ergodic.

For any $f \in L^{1}(Y, \mu)$ the function $1 \otimes f \in L^{1}(\mathcal{R} \times Y, \nu \times \mu)$. Therefore, in view of the maximal inequality for $\eta_{Y}$, there exists an absolute constant $D>0$ so that the following holds. Let $f \in L^{1}(Y, \mu)$; for any $c>0$ we have

$$
\nu \times \mu\left\{(\theta, y) \in \mathcal{R} \times Y: \sup _{n} \frac{1}{n} \sum_{\ell=1}^{n} 1 \otimes f\left(\eta_{Y}^{\ell}(\theta, y)\right) \geq c\right\} \leq \frac{D\|f\|_{1}}{c} .
$$


We also need a maximal inequality similar to and more general than Kolmogorov's inequality in the context of the law of large numbers - see also [2, §3].

Let $\tau>0$ and define an averaging operator $A_{\tau}$ on the space of Borel functions on $Y$ by

$$
A_{\tau} \varphi(y)=\frac{1}{2 \pi} \int_{0}^{2 \pi} \varphi\left(a_{\tau} r_{\theta} y\right) \mathrm{d} \theta
$$

Consider the space $\mathcal{W}=Y^{\mathbb{N}}$ and let $\omega_{y}$ be the Markov measure associated to $A_{\tau}$ and $y$. That is: for bounded Borel function $\phi_{0}, \ldots, \phi_{m}$ on $Y$ we have

$$
\int \phi_{1}\left(w_{1}\right) \cdots \phi_{m}\left(w_{m}\right) \mathrm{d} \omega_{y}(w)=\left(\phi_{1} A_{\tau}\left(\cdots\left(\phi_{m-1} A_{\tau}\left(\phi_{m}\right)\right) \cdots\right)\right)(y)
$$

where $w=\left(\cdots, w_{-1}, w_{1}, w_{2}, \ldots\right)$.

The main case of interest to us is the trajectories obtained using the operator $A_{\tau}$. That is: trajectories of the form

$$
\left(\left(w_{j}\right)_{j \in \mathbb{Z}}\right)=\left(\left(w_{j}\right)_{j \leq 0},\left(a_{\tau} r_{\theta_{j}} \cdots a_{\tau} \theta_{1} w_{0}\right)_{j \geq 1}\right)
$$

for a random $\left(\theta_{j}\right) \in \mathcal{R}$. Let $z \in Y$ we let $\mathcal{W}_{z}$ be the space of all paths as in (5.2) with $w_{0}=z$. In this case, the measure $\omega_{z}$ is obtained by pushing forward $\nu$ to $\mathcal{W}_{z}$.

Fix some $z \in Y$. Let $\rho: \Gamma \rightarrow \mathrm{PGL}_{2}(\mathfrak{l})$ be a representation as in Lemma 5.1. For every $n \geq 1$ define $u_{n, z}: \mathcal{R} \rightarrow \mathrm{PGL}_{2}(\mathfrak{l})$ by

$$
u_{z, n}\left(\left(\theta_{j}\right)\right)=u\left(\tau, r_{\theta_{n}} w_{n-1}\right) \cdots u\left(\tau, r_{\theta_{2}} w_{1}\right) u\left(\tau, r_{\theta_{1}} z\right) ;
$$

where $w_{1}=a_{\tau} r_{\theta_{1}} z$ and for all $j>1$ we have $w_{j}=a_{\tau} r_{\theta_{j}} w_{j-1}$, see (5.2).

Fix some $z \in Y$. For all $\left(\theta_{j}\right) \in \mathcal{R}$ and all $n \geq 1$, define $\phi_{\theta, n}: Y \rightarrow \mathbb{R}$ by

$$
\phi_{\theta, n}(y)=\mathrm{d}_{\mathcal{T}}\left(u(\tau, y) u_{z, n-1}\left(\left(\theta_{j}\right)\right), o\right)-\mathrm{d}_{\mathcal{T}}\left(u_{z, n-1}\left(\left(\theta_{j}\right)\right), o\right) .
$$

In view of (4.6), there is some $L=L(\rho, \tau)$, but independent of $Y$, so that

$$
\left|\phi_{\theta, n}\right| \leq L \text { for all } \theta \text { and } n \text {. }
$$

$$
\text { Put } \varphi_{n}\left(\left(\theta_{j}\right)\right):=\phi_{\theta, n}\left(r_{\theta_{n}} w_{n-1}\right)-\frac{1}{2 \pi} \int_{0}^{2 \pi} \phi_{\theta, n}\left(r_{\theta} w_{n-1}\right) \mathrm{d} \theta \text {. }
$$

5.4. Lemma. For every $c>0$ and $\delta>0$, there exists some $N_{1}=N_{1}(c, \delta, L)$ with the following property. Let $z \in Y$ and define $\varphi_{\ell}$ as above. Then

$$
\nu\left(\left\{\theta=\left(\theta_{j}\right) \in \mathcal{R}: \max _{n \geq N_{1}} \frac{1}{n}\left|\sum_{\ell=1}^{n} \varphi_{\ell}(\theta)\right|>c\right\}\right) \leq \delta .
$$

Proof. This lemma is proved using the following maximal inequality which follows, e.g. by combining [18, p. 386] with [8, Thm 1.1], see also [17, 4].

Let $(\Omega, \mathcal{B}, \beta)$ be a standard probability space and let $\left\{\zeta_{n}\right\}$ be a sequence of bounded Borel functions on $\Omega$ so that $\mathbb{E}_{\beta}\left(\zeta_{n} \mid \zeta_{n-1}, \ldots, \zeta_{1}\right)=0$ for every $n$. 
Then for every $N_{1} \geq 1$ and every $c>0$ we have

$$
\begin{aligned}
\beta\left(\left\{\omega \in \Omega: \max _{N_{1} \leq n \leq N} \frac{1}{n}\left|\sum_{\ell=1}^{n} \zeta_{\ell}(\omega)\right|>c\right\}\right) & \leq \\
& \frac{1}{c^{2}}\left(\sum_{n=N_{1}}^{N} \frac{\int \zeta_{n}^{2}}{n^{2}}+\frac{1}{N_{1}^{2}} \sum_{n=1}^{N_{1}} \int \zeta_{n}^{2}\right) .
\end{aligned}
$$

Returning to our setup, we now observe that

$$
\mathbb{E}_{\nu}\left(\varphi_{n} \mid \varphi_{n-1}, \ldots, \varphi_{1}\right)=\mathbb{E}_{\nu}\left(\mathbb{E}_{\nu}\left(\varphi_{n} \mid \theta_{n-1}, \ldots, \theta_{1}\right) \mid \varphi_{n-1}, \ldots, \varphi_{1}\right) .
$$

Moreover, we have $\mathbb{E}_{\nu}\left(\varphi_{n} \mid \theta_{n-1}, \ldots, \theta_{1}\right)=0$. Hence

$$
\mathbb{E}_{\nu}\left(\varphi_{n} \mid \varphi_{n-1}, \ldots, \varphi_{1}\right)=0 \text {. }
$$

Therefore, we may apply (5.6) with the space $\left(\mathcal{R}, \mathcal{B}^{\otimes \mathbb{Z}}, \nu\right)$ and the sequence $\left\{\varphi_{n}\right\}$ of functions. Since $\int \varphi_{n}^{2} \leq 2 L^{2}$, see (5.5), and $\sum \frac{1}{n^{2}}$ is a convergent series, the lemma follows.

5.5. Conclusion of the proof of Lemma 5.1. Let $0<\varepsilon<1 / 2$ with $L_{1}=<0.01 \lambda_{1}$, see (4.6), and let $\tau=N_{0}+1$ where $N_{0}$ is as in Lemma 4.4.

Let $\delta>0$ be small enough so that for any $g, g^{\prime} \in G$ with $g=h g^{\prime}$ and $\|h-I\| \leq \delta$, we have

$$
\operatorname{dist}\left(a_{\ell} r_{\theta} g, a_{\ell} r_{\theta} g^{\prime}\right)<\alpha_{0} / 2
$$

for all $\theta \in[0,2 \pi]$ and all $0<\ell<2 \tau$, see (4.5).

Let $\mathcal{N}$ denote the $\delta$-neighborhood of $F_{\varepsilon}\left(\tau, \alpha_{0}\right)$ in $X$. In view of (5.7) and Lemma 4.5(2) we have the following. Let $\xi=\left\{\xi_{t}\right\} \subset \mathcal{T}$ with $\xi_{0}=o$ be a geodesic. Then

$$
\frac{1}{2 \pi} \int_{0}^{2 \pi} \mathrm{d} \mathcal{T}\left(u\left(\tau, r_{\theta} x\right), \xi_{t}\right) \mathrm{d} \theta>t+\tau \lambda_{1} / 5
$$

for all $x \in \mathcal{N}$. Let us write $\lambda_{2}:=\lambda_{1} / 5$.

By Theorem [5.2, there exists some $i_{0}$ so that for all $i>i_{0}$ we have

$$
\mu_{H x_{i}}\left(\mathcal{N} \cap Y_{i}\right) \geq 1-2 \varepsilon^{4} .
$$

Fix some $i>i_{0}$ and let $Y=Y_{i}$ and $\mu=\mu_{H x_{i}}$.

By Fubini's theorem, there exists a compact subset $Y^{\prime} \subset \mathcal{N} \cap Y$ with $\mu\left(Y^{\prime}\right) \geq 1-\varepsilon^{3}$ so that for all $y \in Y^{\prime}$, we have

$$
\left|\left\{\theta \in[0,2 \pi]: r_{\theta} y \in \mathcal{N} \cap Y\right\}\right| \geq 1-\varepsilon .
$$

Apply the maximal inequality in (5.1) with $Y, f=\mathbb{1}_{Y \backslash Y^{\prime}}$, and $\tau$ as above. In consequence, there exists an absolute constant $D>0$ so that

$$
\nu \times \mu\left\{(\theta, y) \in \mathcal{R} \times Y: \sup _{n} \frac{1}{n} \sum_{\ell=1}^{n} 1 \otimes f\left(\eta^{\ell}(\theta, y)\right) \geq \varepsilon\right\} \leq D \varepsilon^{2} .
$$


Therefore, if $\varepsilon$ is small enough, Fubini's theorem implies the following: there exists some $Z^{\prime} \subset Y$ with $\mu\left(Z^{\prime}\right)>1-2 \varepsilon$ so that for every $z \in Z^{\prime}$ we have

$$
\nu\left(\left\{\theta \in \mathcal{R}: \sup _{n} \frac{1}{n} \sum_{\ell=1}^{n} 1 \otimes f\left(\eta_{Y}^{\ell}(\theta, z)\right) \geq \varepsilon\right\}\right) \leq \varepsilon .
$$

Let $\theta=\left(\theta_{j}\right)$ be in the complement of the set on the left side of (5.10) and let $\left(w_{j}\right) \in \mathcal{W}_{z}$ be the path obtain from this $\theta$, see (5.2). Then

$$
\frac{1}{n} \sum_{\ell=1}^{n} \mathbb{1}_{Y^{\prime}}\left(w_{\ell}\right) \geq 1-\varepsilon
$$

Put $I_{n}(\theta):=\left\{1 \leq \ell \leq n: w_{\ell} \in Y^{\prime}\right\}$ and $I_{n}^{\prime}(\theta):=\{1 \leq \ell \leq n\} \backslash I_{n}(\theta)$. Then, by (5.11), we have

$$
\# I_{n}(\theta) \geq(1-\varepsilon) n \text {. }
$$

Apply now Lemma 5.4 with this $z \in Z^{\prime}$ and with $c=0.1 \lambda_{2}$ and $\delta=\varepsilon$. Let $N_{1}$ be as in Lemma 5.4 for these choices. Then

$$
\nu\left(\left\{\theta \in \mathcal{R}: \max _{n \geq N_{1}} \frac{1}{n}\left|\sum_{\ell=1}^{n} \varphi_{\ell}(\theta)\right|>0.1 \lambda_{2}\right\}\right) \leq \varepsilon .
$$

Let $\mathcal{R}_{z} \subset \mathcal{R}$ be the compliment of the union of sets appearing on the left sides of (5.10) and (5.13). Let $\theta \in \mathcal{R}_{z}$ and write $I_{n}$ and $I_{n}^{\prime}$ for $I_{n}(\theta)$ and $I_{n}^{\prime}(\theta)$, respectively. Let $\ell \in I_{n}$. Then

$$
w_{\ell}=a_{\tau} \theta_{\ell-1} \cdots a_{\tau} r_{\theta_{1}} z \in Y^{\prime},
$$

and by the definition of $Y^{\prime}, r_{\beta} w_{\ell} \in Y \cap \mathcal{N}$ for some $\beta \in[0,2 \pi]$.

Apply (5.8) with $g=r_{\beta} w_{\ell}$ and the geodesic segment $\xi$ connecting $o$ to $q:=u_{\ell}\left(\left(\theta_{j}, w_{j}\right)\right)$, see (5.3). Let us put $t=\mathrm{d}_{\mathcal{T}}(q, o)$ and parametrize so that $\xi(t)=o$. In consequence, we have the following:

$$
\begin{array}{rlrl}
t+\lambda_{2} \tau & <\frac{1}{2 \pi} \int_{0}^{2 \pi} \mathrm{d}_{\mathcal{T}}\left(u\left(\tau, r_{\theta} r_{\beta} w_{\ell}\right) q, \xi(t)\right) \mathrm{d} \theta & \\
& =\frac{1}{2 \pi} \int_{0}^{2 \pi} \mathrm{d}_{\mathcal{T}}\left(u\left(\tau, r_{\theta} w_{\ell}\right) q, o\right) \mathrm{d} \theta \quad o=\xi(t) .
\end{array}
$$

Moreover, by (5.4) we have

$$
\frac{1}{2 \pi} \int_{0}^{2 \pi} \phi_{\theta, \ell+1}\left(r_{\theta} w_{\ell}\right) \mathrm{d} \theta=\frac{1}{2 \pi} \int_{0}^{2 \pi} \mathrm{d}_{\mathcal{T}}\left(u\left(\tau, r_{\theta} w_{\ell}\right) q, o\right)-\mathrm{d}_{\mathcal{T}}(q, o) \mathrm{d} \theta .
$$

Recall that $\mathrm{d}_{\mathcal{T}}(q, o)=t$, therefore, using the above and (5.14) we get that

$$
\frac{1}{2 \pi} \int_{0}^{2 \pi} \phi_{\theta, \ell+1}\left(r_{\theta} w_{\ell}\right) \mathrm{d} \theta \geq \lambda_{2} \tau \quad \text { if } \ell \in I_{n}
$$


Recall further from (4.6) that $\mathrm{d}_{\mathcal{T}}(u(\tau, g), e) \leq L_{1}$ t for all $g \in F$; thus, we may use the triangle inequality and get also the trivial estimate

$$
\left|\frac{1}{2 \pi} \int_{0}^{2 \pi} \phi_{\theta, \ell+1}\left(r_{\theta} w_{\ell}\right) \mathrm{d} \theta\right| \leq \frac{1}{2 \pi} \int_{0}^{2 \pi}\left|\mathrm{d}_{\mathcal{T}}\left(u\left(\tau, r_{\theta} w_{\ell}\right) q, o\right)-\mathrm{d}_{\mathcal{T}}(q, o)\right| \mathrm{d} \theta
$$

$$
\leq L_{1}
$$

for all $0 \leq \ell \leq n-1$.

In view of (5.15) and (5.16), for every $n \in \mathbb{N}$ we have

$$
\begin{aligned}
\sum_{\ell=1}^{n} \frac{1}{2 \pi} \int_{0}^{2 \pi} \phi_{w, \ell}\left(r_{\theta} w_{\ell-1}\right) \mathrm{d} \theta & \geq\left(\# I_{n}\right) \lambda_{2} \tau-\left(n-\# I_{n} \sqrt{L_{1} \tau}\right. \\
\varepsilon<0.1 \&\left[L_{1} k 0.1 \lambda_{2} \rightsquigarrow\right. & \geq(1-\varepsilon) \lambda_{2} \tau n-\lambda_{2} \tau n / 2
\end{aligned}
$$

Let now $n>N_{1}$. Therefore, since $\left(\left(\theta_{j}, w_{j}\right)\right) \in \mathcal{R}_{z}$, we conclude from (5.13) that

$$
\frac{1}{n}\left|\sum_{\ell=1}^{n} \varphi_{\ell}(\theta)\right| \leq 0.1 \lambda_{2} .
$$

Recall again from (5.4) the definition of $\phi_{\theta, n}\left(r_{\theta_{n}} w_{n-1}\right)$, also recall that $\varphi_{n}=$ $\phi_{\theta, n}-\frac{1}{2 \pi} \int_{0}^{2 \pi} \phi_{\theta, n}$. We thus obtain

$$
\sum_{\ell=1}^{n} \varphi_{\ell}(\theta)=\mathrm{d}_{\mathcal{T}}\left(u_{z, n}(\theta), o\right)-\sum_{\ell=1}^{n} \frac{1}{2 \pi} \int_{0}^{2 \pi} \phi_{\theta, \ell}\left(r_{\theta} w_{\ell-1}\right) \mathrm{d} \theta .
$$

This, together with (5.18) and (5.17), implies that for all $n \geq N_{1}$ we have

$$
\mathrm{d}_{\mathcal{T}}\left(u_{z, n}(\theta), o\right) \geq(\tau / 2-1 / 10) \lambda_{2} n \geq \lambda_{2} \tau n / 3=\lambda_{1} \tau n / 15 .
$$

To get Lemma 5.1 from (5.19) it remains to note that trajectories

$$
\left\{a_{\tau} \theta_{n} \cdots a_{\tau} r_{\theta_{1}} z:\left(\theta_{j}\right) \in \mathcal{R}\right\}
$$

give rise to the rotation invariant distribution on the boundary circle corresponding to $H g_{z}$, recall that $g_{z} \in F$. Moreover, for $\nu$-a.e. $\theta=\left(\theta_{j}\right) \in \mathcal{R}$ there exists a unique geodesic $\left\{\xi_{\theta, t}\right\}$ with $\xi_{\theta, 0}=g_{z}$ so that the trajectory $a_{\tau} \theta_{n} \cdots a_{\tau} r_{\theta_{1}} g_{z}$ is at a sublinear distance from $\left\{\xi_{\theta, t}\right\}$, see Lemma 2.4. Indeed even a central limit theorem holds for these trajectories [11, 10, 16, 3.

\section{Proof of Proposition 3.1}

In this section we complete the proof of Proposition 3.1. The proof uses Lemma [5.1, we begin with some preliminary statements.

Let the notation be as in Proposition 3.1. In particular, recall that for any $g \in \mathrm{PGL}_{2}\left(\mathfrak{l}_{v}\right), \mathfrak{C}_{g}$ denotes the graph of the linear fractional transformation

$$
g: \mathbb{P l}_{v} \rightarrow \mathbb{P l}_{v} .
$$

Alternatively, $\mathfrak{C}_{g}$ is the image of $\left\{\left(h, g h g^{-1}\right): h \in \mathrm{PGL}_{2}\left(\mathfrak{l}_{v}\right)\right\}$ in $\mathbb{P l}_{v} \times \mathbb{P l}_{v}$. 
Let $\alpha, \beta \in \mathfrak{l}_{v}$ and define

$$
\operatorname{Crs}(\alpha, \beta)=\left\{([\alpha, 1],[s, 1]) \in \mathbb{P l}_{v} \times \mathbb{P l}_{v}\right\} \cup\left\{([r, 1],[\beta, 1]) \in \mathbb{P l}_{v} \times \mathbb{P l}_{v}\right\} .
$$

We refer to $\operatorname{Crs}(\alpha, \beta)$ as crosses.

6.1. Lemma. Let $\left\{g_{i}\right\}$ be a squence of elements in $\mathrm{PGL}_{2}\left(\mathfrak{l}_{v}\right)$. Then at least one of the following holds.

(1) There exists a subsequence $\left\{g_{i_{m}}\right\}$ and some $g \in \mathrm{PGL}_{2}\left(\mathfrak{l}_{v}\right)$ so that $\mathfrak{C}_{g_{i m}} \rightarrow \mathfrak{C}_{g}$, or

(2) there exists a subsequence $\left\{g_{i_{m}}\right\}$ so that $\left\{\mathfrak{C}_{g_{i_{m}}}\right\}$ converges to a cross or a union of a line and a point.

Proof. If there exists a subsequence $\left\{g_{i_{m}}\right\}$ and some $g \in \mathrm{PGL}_{2}\left(\mathfrak{l}_{v}\right)$ so that $g_{i_{m}} \rightarrow g$, then $\mathfrak{C}_{g_{i_{m}}} \rightarrow \mathfrak{C}_{g}$. That is: part (1) holds.

Therefore, we may assume that $g_{i} \rightarrow \infty$ and will show that part (2) holds in this case. Passing to a subsequence we may assume all $g_{i}$ 's are in one $\mathrm{PSL}_{2}\left(\mathfrak{l}_{v}\right)$ coset, hence we assume $\operatorname{det}\left(g_{i}\right)=k$ for all $i$.

We use the projective coordinates, in those coordinates we have: $\mathbb{P l}_{v}=$ $\left\{[r, s]: r, s \in \mathfrak{l}_{v}\right\}$ and

$$
\mathfrak{C}_{g_{i}}=\left\{\left([r, s],\left[a_{i} r+b_{i} s, c_{i} r+d_{i} s\right]\right\}\right.
$$

where $g_{i}=\left(\begin{array}{cc}a_{i} & b_{i} \\ c_{i} & d_{i}\end{array}\right)$.

First let us assume that for all but finally many $g_{i}$ 's we have $c_{i} \neq 0$. Omitting these finitely many terms, we assume $c_{i} \neq 0$. Using the nonhomogeneous coordinates, we have

$$
\begin{aligned}
& \mathfrak{C}_{g_{i}}=\left\{\left([r, 1],\left[\frac{a_{i} r+b_{i}}{c_{i} r+d_{i}}, 1\right]:-d_{i} / c_{i}\right.\right.\left.\neq r \in \mathfrak{l}_{v}\right\} \cup \\
&\left\{\left([1,0],\left[a_{i} / c_{i}, 1\right]\right\} \cup\left\{\left(\left[-d_{i} / c_{i}, 1\right],[1,0]\right)\right\}\right.
\end{aligned}
$$

Alternatively, except for two points, the graph $\mathfrak{C}_{g_{i}}$ is given by the equation

$$
\left(s-a_{i} / c_{i}\right)\left(r+d_{i} / c_{i}\right)=-k / c_{i}^{2}
$$

- recall that $\operatorname{det}\left(g_{i}\right)=k$. The missing two points can be obtained by taking limit as $r \rightarrow \infty$ or $s \rightarrow \infty$.

Now if the sequence $\left\{c_{i}\right\}$ is bounded away from $\infty$ and 0 , then since $g_{i} \rightarrow \infty$ we get from (6.1) that $\mathfrak{C}_{g_{i}}$ converges to $\left\{\left([1,0],\left[-d_{i} / c_{i}, 1\right]\right\}\right.$ or $\left\{\left(\left[a_{i} / c_{i}, 1\right],[1,0]\right)\right\}$. Therefore, we may assume that passing to a subsequence either $c_{i} \rightarrow 0$ or $c_{i} \rightarrow \infty$. In either case we get the conclusion in part (2).

It remains to consider the case where $c_{i_{m}}=0$ along a subsequence. In this case, $\left\{\mathfrak{C}_{g_{i_{m}}}\right\}$ converges to the union of a line and a point.

For $j=1,2$, let

$$
\mathfrak{p}_{j}: \mathrm{PGL}_{2} \times \mathrm{PGL}_{2} \rightarrow \mathrm{PGL}_{2}
$$

be the projection onto the $j$-th component; put $\sigma_{j}=\mathfrak{p}_{j} \circ \sigma: \Gamma \rightarrow \mathrm{PGL}_{2}\left(\mathfrak{l}_{v}\right)$.

If $\sigma(\Gamma) \subset{ }^{\sigma} \mathbf{G}\left(\mathrm{L}_{v}\right)$ is unbounded, then either $\sigma_{1}(\Gamma)$ or $\sigma_{2}(\Gamma)$ is unbounded. Using Lemma 5.1 we can show that indeed both these projections are unbounded: 
6.2. Lemma. Let $M=\mathbb{H}^{3} / \Gamma$ be a finite volume hyperbolic 3-manifold which contains infinitely many totally geodesic surfaces, $\left\{\mathrm{S}_{i}: i \in \mathbb{N}\right\}$. For every $i \geq 1$ let $\Delta_{i} \subset \Gamma$ denote the fundamental group of the surface $S_{i}$. Suppose $\sigma_{j}(\Gamma)$ is unbounded for some $j=1,2$. Then both $\sigma_{1}\left(\Delta_{i}\right)$ and $\sigma_{2}\left(\Delta_{i}\right)$ are unbounded for all large enough $i$. In particular, both $\sigma_{1}(\Gamma)$ and $\sigma_{2}(\Gamma)$ are unbounded.

Proof. For every $i$ let ${ }^{\sigma} \mathbf{H}_{i}$ denote the Zariski closure of $\sigma\left(\Delta_{i}\right)$. Let $j$ be so that $\sigma_{j}(\Gamma)$ is unbounded. Then it follows from Lemma 5.1 that $\sigma_{j}\left(\Delta_{i}\right)$ is unbounded for all large enough $i$. Therefore, by Lemma 2.2 we have: for all large enough $i$, there exists some $g_{i} \in \mathrm{PGL}_{2}\left(\mathfrak{l}_{v}\right)$ so that

$$
{ }^{\sigma} \mathbf{H}_{i}\left(\mathfrak{l}_{v}\right) \cap\left\{\left(h, g_{i} h g_{i}^{-1}\right): h \in \mathrm{PGL}_{2}\left(\mathfrak{l}_{v}\right)\right\}
$$

is a subgroup of index at most 8 in ${ }^{\sigma} \mathbf{H}_{i}\left(\mathfrak{l}_{v}\right)$.

The claims hold for all such $i$.

6.3. The definition of $\Psi$. Recall that $M=\mathbb{H}^{3} / \Gamma$ is a closed hyperbolic 3-manifold containing infinitely many totally geodesic surfaces, $\left\{\mathrm{S}_{i}: i \in \mathbb{N}\right\}$.

For each $i \in \mathbb{N}$, we let $\Delta_{i} \subset \Gamma$ be the fundamental group of $\mathrm{S}_{i}$. Let ${ }^{\sigma} \mathbf{H}_{i} \subset \mathrm{PGL}_{2} \times \mathrm{PGL}_{2}$ denote the Zariski closure of $\sigma\left(\Delta_{i}\right)$ for every $i$.

Let $i$ be large enough so that Lemma 6.2 holds true. In particular, there exists some $g_{v, i} \in \mathrm{PGL}_{2}\left(\mathfrak{l}_{v}\right)$ so that

$$
\left\{\left(h, g_{v, i} h g_{v, i}^{-1}\right): h \in \mathrm{PSL}_{2}\left(\mathfrak{l}_{v}\right)\right\}
$$

has index at most 8 in ${ }^{\sigma} \mathbf{H}_{i}\left(\mathfrak{l}_{v}\right)$. Let $\mathfrak{C}_{i}$ denote the image of ${ }^{\sigma} \mathbf{H}_{i}\left(\mathfrak{l}_{v}\right)$ in $\mathbb{P l}_{v} \times \mathbb{P l}_{v}$; as was mentioned before, $\mathfrak{C}_{i}$ equals the image of

$$
\left\{\left(h, g_{v, i} h g_{v, i}^{-1}\right): h \in \mathrm{PGL}_{2}\left(\mathfrak{l}_{v}\right)\right\}
$$

in $\mathbb{P l}_{v} \times \mathbb{P l}_{v}$.

Recall that each $\mathrm{S}_{i}$ gives rise to a periodic $H$-orbits, $H x_{i}$. For every $i$, the orbit $H x_{i}$ corresponds to a closed $\Gamma$-orbit

$$
\mathcal{C}_{i}=\left\{C_{i} \Gamma\right\} \subset \mathcal{C} .
$$

Identify $X$ with $F$, then $H x_{i}$ is identified with a subset $Y_{i} \subset F$; note that $Y_{i}$ has only finitely many connected components. For every $y \in H x_{i}$ we let $g_{y} \in F$ be the point corresponding to $y$. The orbit $H g_{y}$ gives rise to a plane $P_{y}$ in $\mathbb{H}^{3}$ and a circle

$$
C_{y}:=\partial P_{y}=C_{i} \gamma_{y} \in \mathcal{C}_{i} \text { for some } \gamma \in \Gamma .
$$

By Lemma 6.2. $\sigma_{j}(\Gamma)$ is unbounded for $j=1,2$. For $j=1,2$, let $u_{j}$ be the cocycle and $\psi_{j}$ the equivariant map constructed in 4.1 using the representation $\sigma_{j}$. Define

$$
\Psi:=\left(\psi_{1}, \psi_{2}\right) .
$$

We will show that $\Psi$ satisfies the claims in Proposition 3.1 .

6.4. Lemma. Let $\varepsilon>0$. There exist

- natural numbers $N=N(\varepsilon)$ and $\tau=\tau(\varepsilon)$, 
- for every $i>N$, a subset $Z_{i}^{\prime} \subset H x_{i}$ with $\mu_{H x_{i}}\left(Z_{i}^{\prime}\right)>1-\varepsilon$, and

- for every $z \in Z_{i}^{\prime}$, a subset $R_{z} \subset[0,2 \pi]$ with $\left|R_{z}\right|>2(1-\varepsilon) \pi$ so that the following hold.

(1) For every $z \in Z_{i}^{\prime}, \theta \in R_{z}, n>N_{0}$, and $j=1,2$ we have

$$
\mathrm{d}_{\mathcal{T}}\left(u_{j}\left(n \tau, r_{\theta} z\right), o\right)>\lambda_{0} \tau n .
$$

(2) For every $z \in Z_{i}^{\prime}$ and $\theta \in R_{z}$ put

$$
\beta_{g_{z}, \theta}:=\lim _{n} a_{n \tau} r_{\theta} g_{z} \in C_{z}=C_{i} \gamma_{z}
$$

where $g_{z}, C_{z}$, and $\gamma_{z}$ are as in (6.2). Then

$$
\Psi\left(\beta_{g_{z}, \theta}\right) \in \mathfrak{C}_{i} \sigma\left(\gamma_{z}\right) .
$$

Proof. By Lemma 6.2, $\sigma_{j}(\Gamma)$ is unbounded for $j=1,2$. Let

$$
i_{0}=i_{0}\left(\varepsilon^{2} / 2\right), \tau=\tau\left(\varepsilon^{2} / 2\right), \text { and } N=N\left(\varepsilon^{2} / 2\right)
$$

be so that the conclusion of Lemma 5.1 holds with $\varepsilon^{2} / 2$ and both $\sigma_{1}$ and $\sigma_{2}$. Moreover, assume that Lemma 6.2 holds true for all $i>i_{0}$.

Let $Z_{i} \subset Y_{i}$ be a subset with $\mu_{H x_{i}}\left(Z_{i}\right)>1-\varepsilon^{2}$ so that the conclusion of Lemma 5.1 holds for all $z \in Z_{i}$ and for both $\sigma_{1}$ and $\sigma_{2}$.

For every $z \in Z_{i}$, let

$$
R_{z}=\left\{\theta \in[0,2 \pi]: r_{\theta} z \in Z_{i}\right\} .
$$

By Fubini's theorem there is a subset $Z_{i}^{\prime} \subset Z_{i}$ with $\mu_{H x_{i}}\left(Z_{i}^{\prime}\right)>1-\varepsilon$ so that for all $z \in Z_{i}^{\prime}$ we have $\left|R_{z}\right|>2(1-\varepsilon) \pi$.

We will show that the lemma holds with this $N, Z_{i}^{\prime}$, and $R_{z}$. Let $z \in Z_{i}^{\prime}$. Then by Lemma 5.1 we have

$$
\mathrm{d}_{\mathcal{T}}\left(u_{j}\left(n \tau, r_{\theta} z\right), o\right)>\lambda_{0} \tau n, \quad \theta \in R_{z}, n>N, \text { and } j=1,2 .
$$

This establishes the part (1).

Let $\theta \in R_{z}$. Then by (6.4) and Lemma 2.4 we have the following. There exists a unique geodesic $\left\{\xi_{t}: t \in \mathbb{R}\right\} \subset \mathcal{T}$ with $\xi_{0}=o$ so that

$$
u_{j}\left(n \tau, r_{\theta} z\right) \rightarrow \xi_{\infty} \in \partial \mathcal{T} \text {. }
$$

Moreover, for every $\eta>0$ there is some $\left.N^{\prime}=N^{\prime} L_{1}, \lambda_{0}, \tau_{0}, N_{0}, \eta\right)$ so that

$$
\mathrm{d}_{\mathcal{T}}\left(u_{j}\left(n \tau, r_{\theta} z\right),\left\{\xi_{t}\right\}\right) \leq \eta n \tau \quad \text { for all } n>N^{\prime} \text { and } j=1,2 .
$$

Note also that $a_{t} r_{\theta} \in H$ for all $t$ and $\theta$. Therefore, $\left\{a_{n \tau_{0}} r_{\theta} g_{z}: n \in \mathbb{Z}\right\} \subset$ $P_{z}$. Moreover, since $H g_{z} \Gamma$ is closed, we get the following:

$$
u_{j}\left(n_{m} \tau, r_{\theta} z\right) \in \mathcal{T}^{\prime} \text { for a sequence } n_{m} \rightarrow \infty
$$

where $\mathcal{T}^{\prime}$ is the subtree corresponding to $\sigma(\gamma)^{-1} \mathbf{H}_{i} \sigma(\gamma)$.

Since by (6.7) we have $u_{j}\left(n_{m} \tau, r_{\theta} z\right) \rightarrow \xi_{\infty}$, the definition of $\Psi$, (6.3), and (6.9) imply that $\Psi\left(\beta_{g_{z}, \theta}\right) \in \partial \mathcal{T}^{\prime}=\mathfrak{C}_{i} \sigma\left(\gamma_{z}\right)$.

This finishes the proof of part (2) and the lemma.

Recall that for a circle $C$ we denote the length measure on $C$ by $\mathrm{m}_{C}$. The following is a crucial step in the proof Proposition 3.1. 
6.5. Lemma. For every $\varepsilon>0$ there exists a subset $\hat{F}_{\varepsilon} \subset F$ with

$$
\operatorname{vol}_{X}\left(\hat{F}_{\varepsilon}\right)>1-4 \varepsilon
$$

and for every $g \in \hat{F}_{\varepsilon}$ a subset $\hat{C}_{g, \varepsilon} \subset C_{g}$ with $\mathrm{m}_{C_{g}}\left(\hat{C}_{g, \varepsilon}\right) \geq(1-2 \varepsilon) \mathrm{m}_{C_{g}}\left(C_{g}\right)$ so that one of the the following holds.

(1) There exists some $h_{g} \in \mathrm{PGL}_{2}\left(\mathfrak{l}_{v}\right)$ so that $\Psi\left(\hat{C}_{g, \varepsilon}\right) \subset \mathfrak{C}_{h_{g}}$, or

(2) $\Psi\left(\hat{C}_{g, \varepsilon}\right)$ is contained in a cross or the union of a line and a point.

Proof. Fix some $\varepsilon>0$. Let $i_{0}, N, \tau, Z_{i}^{\prime}$, and $R_{z}$ be as in Lemma 6.4 applied with this $\varepsilon$.

Let $F_{\varepsilon}$ and $N_{0}$ be as in Lemma 4.4. Fix a geodesic $\left\{\xi_{t}\right\}$ emanating from $o$ for the rest of the proof. Let $R_{g} \subset[0,2 \pi]$ be the set where Lemma 4.4 holds for $\left\{\xi_{t}\right\}, g, u_{1}$, and $u_{2}$. In particular, $\left|R_{g}\right|>2(1-\varepsilon) \pi$ and for $j=1,2$ we have

$$
\mathrm{d}_{\mathcal{T}}\left(u_{j}\left(n, r_{\theta} g\right), o\right)>\lambda_{1} n / 3 \quad \text { for all } \theta \in R_{g}, n>N_{0} \text {, and } t \in \mathbb{R} .
$$

For any $g \in F_{\varepsilon}$, let $P_{g}$ be the plane corresponding to $H g$; put $C_{g}=\partial P_{g}$. For any $\theta \in R_{g}$, define

$$
\beta_{g, \theta}:=\lim _{n} a_{n \tau} r_{\theta} g \in C_{g}
$$

Then $\Psi\left(\beta_{g, \theta}\right) \in \Psi\left(C_{g}\right)$.

Given $i$ and $\delta>0$, let $\mathcal{N}_{i, \delta}$ be the $\delta$-neighborhood of $Z_{i}^{\prime}$. By Corollary 5.2.1, there exists some $i_{1}(\delta)$ so that $\operatorname{vol}_{X}\left(\mathcal{N}_{i, \delta}\right)>1-3 \varepsilon$ for all $i>i_{1}(\delta)$.

Claim. For every $\eta>0$, there exists some $\delta>0$ so that the following holds. Let $i>i_{\eta}:=\max \left\{i_{0}, i_{1}(\delta)\right\}$ and let $g \in F_{\varepsilon} \cap \mathcal{N}_{i, \delta}$. Then there exists some $C_{g, \eta}^{\prime} \subset C_{g}$ with

$$
\mathrm{m}_{C_{g}}\left(C_{g, \eta}^{\prime}\right)>(1-3 \varepsilon) \mathrm{m}_{C_{g}}\left(C_{g}\right)
$$

so that $\Psi\left(C_{g, \eta}^{\prime}\right)$ lies in the $\eta$-neighborhood of $\mathfrak{C}_{h}$ for some $h \in \mathrm{PGL}_{2}\left(\mathfrak{l}_{v}\right)$.

Let us first assume the claim and finish the proof of the lemma. For every $m \in \mathbb{N}$, let $\eta_{m}=1 / m$ and let $\delta_{m}$ and $i_{m}$ be given by applying the claim with $\eta_{m}$. Set

$$
\hat{F}_{\varepsilon}:=F_{\varepsilon} \cap\left(\bigcap_{\ell \geq 1} \bigcup_{m \geq \ell} \mathcal{N}_{i_{m}, \delta_{m}}\right) ;
$$

note that $\operatorname{vol}_{X}\left(\hat{F}_{\varepsilon}\right) \geq 1-4 \varepsilon$.

Let $g \in \hat{F}_{\varepsilon}$ and let $C_{g}$ be the corresponding circle. Then there exists a subsequence $\left\{m_{k}\right\}$ so that $g \in \mathcal{N}_{i_{m_{k}}, \delta_{m_{k}}}$. In view of the claim, for every $k$, there exists some $C_{g, m_{k}}^{\prime} \subset C_{g}$ and some $h_{k} \in \mathrm{PGL}_{2}\left(\mathfrak{l}_{v}\right)$ with $\mathrm{m}_{C_{g}}\left(C_{g, m_{k}}^{\prime}\right)>$ $(1-2 \varepsilon) \mathrm{m}_{C_{g}}\left(C_{g}\right)$ so that

$$
\Psi\left(C_{g, m_{k}}^{\prime}\right) \text { lies in the } 1 / m_{k} \text {-neighborhood of } \mathfrak{C}_{h_{k}} .
$$

Apply Lemma 6.1 with the sequence $\left\{\mathfrak{C}_{h_{k}}: k \in \mathbb{N}\right\}$. Then there exists a subsequence $\left\{k_{i}\right\}$ so that one of the following holds.

(1) $\lim _{i} \mathfrak{C}_{h_{k_{i}}}=\mathfrak{C}_{h}$ for some $h \in \mathrm{PGL}_{2}\left(\mathfrak{l}_{v}\right)$, or 
(2) $\lim _{i} \mathfrak{C}_{h_{k_{i}}}$ is contained in a cross or the union of a line and a point.

Let

$$
\hat{C}_{g, \varepsilon}:=\bigcap_{\ell \geq 1} \bigcup_{i \geq \ell} C_{g_{k_{i}}, m_{k_{i}}}^{\prime}
$$

then $\mathrm{m}_{C}\left(\hat{C}_{g, \varepsilon}\right)>(1-2 \varepsilon) \mathrm{m}\left(C_{g}\right)$. Moreover, for every $\beta \in \hat{C}_{g, \varepsilon}$ we have $\Psi(\beta) \in \lim _{i} \mathfrak{C}_{h_{k_{i}}}$. The lemma follows.

Proof of the claim. Let $g \in F_{\varepsilon}$. Recall that we fixed a geodesic $\left\{\xi_{t}\right\}$ emanating from $o$, and let $R_{g} \subset[0,2 \pi]$ be the set where Lemma 4.4 holds for $\left\{\xi_{t}\right\}, g, u_{1}$, and $u_{2}$. For $g \in F_{\varepsilon}$ and $\theta \in R_{g}$, define

$$
u\left(n \tau, r_{\theta} g\right)=\left(u_{1}\left(n \tau, r_{\theta} g\right), u_{2}\left(n \tau, r_{\theta} g\right)\right) .
$$

For any $z \in Z_{i}^{\prime}$, let $R_{z}$ be as in Lemma 6.4. Similarly, for all $i>i_{0}$, $z \in Z_{i}^{\prime}$, and $\theta \in R_{z}$ define

$$
u\left(n \tau, r_{\theta} g_{z}\right)=\left(u_{1}\left(n \tau, r_{\theta} g_{z}\right), u_{2}\left(n \tau, r_{\theta} g_{z}\right)\right) .
$$

Thanks to (6.4), (6.10), and Lemma 2.4, there exists some $n_{\eta}$ so that for all $n \geq n_{\eta}$ we have $u\left(n \tau, r_{\theta} g_{z}\right)$ and $u\left(n \tau, r_{\theta} g\right)$ approximate $\Psi\left(\beta_{g_{z}, \theta}\right)$ and $\Psi\left(\beta_{g, \theta}\right)$, respectively, within $\eta / 4$.

Let $n=n_{\eta}$ and let $\delta>0$ be so that if $\mathrm{d}\left(h_{1}, h_{2}\right)<\delta$ for $h_{1}, h_{2} \in G$, then $\mathrm{d}\left(a_{n \tau} h_{1}, a_{n \tau} h_{2}\right) \leq \eta / 4$ where d denotes the right invariant Riemannian metric on $G$.

Apply Corollary 5.2.1 with the sets $\mathcal{N}_{i, \delta}$ and let $i_{1}(\delta)$ be as in that Corollary. Let $i>\max \left\{i_{0}, i_{1}(\delta)\right\}$ and let $g \in F_{\varepsilon} \cap \mathcal{N}_{i, \delta}$. Then there exists some $g_{z} \in Z_{i}^{\prime}$ so that $\mathrm{d}\left(g, g_{z}\right)<\delta$.

The claim thus holds with $C_{g, \eta}^{\prime}=\left\{\beta_{g, \theta}: \theta \in R_{z} \cap R_{g}\right\}$.

Proof of Proposition 3.1. First note that in view of Lemma 2.7, $\Psi$ satisfies part (1) in the proposition - recall that $\mathrm{PGL}_{2} \times \mathrm{PGL}_{2}$ acts transitively on $\mathbb{P l}_{v} \times \mathbb{P l}_{v}$,

We now show that $\Psi$ also satisfies part (2) in the proposition. We claim that there exists a full measure subset $\hat{F} \subset F$, and for every $g \in \hat{F}$ a full measure subset $\hat{C}_{g} \subset C_{g}$ so that one of the following holds.

(1) There exists some $h_{g} \in \mathrm{PGL}_{2}\left(\mathfrak{l}_{v}\right)$ so that $\Psi\left(\hat{C}_{g}\right) \subset \mathfrak{C}_{h_{g}}$, or

(2) $\Psi\left(\hat{C}_{g}\right)$ is contained in a cross or the union of a line and a point.

Apply Lemma 6.5 with $\varepsilon=1 / m$ for all $m \in \mathbb{N}$. Let $\hat{F}_{m}=\hat{F}_{1 / m}$ and for every $g \in \hat{F}_{m}$ let $\hat{C}_{m, g}=\hat{C}_{1 / m, g}$ denote the sets obtained by that lemma. Define

$$
\hat{F}=\bigcap_{\ell \geq 1} \bigcup_{m \geq \ell} \hat{F}_{m}
$$

Then $\hat{F}$ is conull in $F$.

Moreover, for every $g \in \hat{F}$ there exists a subsequence $m_{k}$ so that $g \in \hat{F}_{m_{k}}$ for all $k$. Let $\hat{C}_{g}=\bigcap_{\ell \geq 1} \bigcup_{k \geq \ell} \hat{C}_{m_{k}, g}$. Then $\hat{C}_{g} \subset C_{g}$ is conull in $C_{g}$. 
Moreover, $\hat{C}_{g}$ satisfies (1) or (2) in the claim - recall that the same property holds for $\hat{C}_{m_{k}, g}$ by Lemma 6.5 .

We now show that (1) above holds almost surely. To see this, set $\mathcal{L}_{1}:=$ $\left\{\mathfrak{C}_{h}: h \in \mathrm{PGL}_{2}\left(\mathfrak{l}_{v}\right)\right\}$ and $\mathcal{L}_{2}:=$ union of a line and a point or crosses $\}$. Note that both $\mathcal{L}_{1}$ and $\mathcal{L}_{2}$ are $\Gamma$-invariant. Moreover, $\Psi$ is $\Gamma$-equivariant and $\Gamma$ acts ergodically on $\mathcal{C}$. Therefore, either the essential image of $\left.\Psi\right|_{C}$ belongs to $\mathcal{L}_{1}$ a.e. $C \in \mathcal{C}$ or the essential image of $\left.\Psi\right|_{C}$ belongs to $\mathcal{L}_{2}$ a.e. $C \in \mathcal{C}$.

Let $\xi \neq \xi^{\prime} \in \mathbb{S}^{2}$ be so that $\Psi(\xi)=([r, 1],[s, 1])$ and $\Psi\left(\xi^{\prime}\right)=\left(\left[r^{\prime}, 1\right],\left[s^{\prime}, 1\right]\right)$ with $r \neq r^{\prime}$ and $s \neq s^{\prime}$ - in view of Lemma 2.7 we may find such points. Then there are exactly two crosses passing through both of $\Psi(\xi)$ and $\Psi\left(\xi^{\prime}\right)$; similarly for union of a line and a point. However, the set of circles in $\mathbb{S}^{2}$ passing through $\left\{\xi, \xi^{\prime}\right\}$ covers the entire $\mathbb{S}^{2}$. Therefore, the essential image of $\left.\Psi\right|_{C}$ belongs to $\mathcal{L}_{2}$ a.e. $C \in \mathcal{C}$ would contradict Lemma 2.7 .

In consequence, we have: for a.e. $g \in \hat{F}$ there exists some $h_{g} \in \mathrm{PGL}_{2}\left(\mathfrak{l}_{v}\right)$ so that $\Psi\left(\hat{C}_{g}\right) \subset \mathfrak{C}_{h_{g}}$. Since $\Psi$ is $\Gamma$-equivariant, this concludes the proof of the proposition.

\section{REFERENCES}

1. U. Bader, D. Fisher, N. Miller, and M. Stover, Arithmeticity, superrigidity, and totally geodesic submanifolds, arXiv:1903.08467 (2019).

2. Yves Benoist and Jean-Francois Quint, Stationary measures and invariant subsets of homogeneous spaces (iii), Annals of Mathematics 178 (2013), 1017-1059.

3. Marc A. Berger, Central limit theorem for products of random matrices, Transactions of the American Mathematical Society 285 (1984), no. 2, 777-803.

4. Y. S. Chow, A martingale inequality and the law of large numbers, Proc. Amer. Math. Soc. 11 (1960), 107-111. MR 0112190

5. Kevin Corlette, Archimedean superrigidity and hyperbolic geometry, Annals of Mathematics 135 (1992), no. 1, 165-182.

6. K. Delp, D. Hoffoss, and Manning J.F., Problems in groups, geometry, and threemanifolds, arXiv:1512.04620. (2015).

7. Alex Eskin, Gregory Margulis, and Shahar Mozes, Upper bounds and asymptotics in a quantitative version of the oppenheim conjecture, Annals of Mathematics 147 (1998), no. $1,93-141$.

8. I. Fazekas and O. Klesov, A general approach to the strong laws of large numbers, Teor. Veroyatnost. i Primenen. 45 (2000), no. 3, 568-583. MR 1967791

9. D. Fisher, J.F. Lafont, N. Miller, and M. Stover, Finiteness of maximal geodesic submanifolds in hyperbolic hybrids, arXiv:1802.04619 (2018).

10. H. Furstenberg and H. Kesten, Products of random matrices, Ann. Math. Statist. 31 (1960), no. 2, 457-469.

11. Harry Furstenberg, A poisson formula for semi-simple lie groups, Annals of Mathematics 77 (1963), no. 2, 335-386.

12. Harry Furstenberg, Boundary theory and stochastic processes on homogeneous spaces, Harmonic analysis on homogeneous spaces (Proc. Sympos. Pure Math., Vol. XXVI, Williams Coll., Williamstown, Mass., 1972), Amer. Math. Soc., Providence, R.I., 1973, pp. $193-229$.

13. H. Garland and M. S. Raghunathan, Fundamental domains for lattices in (r-)rank 1 semisimple lie groups, Annals of Mathematics 92 (1970), no. 2, 279-326.

14. I. Ya. Goldsheid and G. A. Margulis, Lyapunov exponents of a product of random matrices, Uspekhi Mat. Nauk 44 (1989), no. 5(269), 13-60. MR 1040268 
15. Mikhail Gromov and Richard Schoen, Harmonic maps into singular spaces andpadic superrigidity for lattices in groups of rank one, Publications Mathématiques de l'Institut des Hautes Études Scientifiques 76 (1992), no. 1, 165-246.

16. A. Guivarc'h, Quelques proprietes asymptotiques des produits de matrices aleatoires, Ecole d'Eté de Probabilités de Saint-Flour VIII-1978 (Berlin, Heidelberg) (P. L. Hennequin, ed.), Springer Berlin Heidelberg, 1980, pp. 177-250.

17. J. Hájek and A. Rényi, Generalization of an inequality of Kolmogorov, Acta Math. Acad. Sci. Hungar. 6 (1955), 281-283. MR 0076207

18. Michel Loève, Probability theory. Foundations. Random sequences, D. Van Nostrand Company, Inc., Toronto-New York-London, 1955. MR 0066573

19. Colin Maclachlan and Alan W. Reid, The arithmetic of hyperbolic 3-manifolds, Graduate Texts in Mathematics, vol. 219, Springer-Verlag, New York, 2003.

20. G. Margulis and A. Mohammadi, Arithmeticity of hyperbolic 3-manifolds containing infinitely many totally geodesic surfaces, arXiv:1902.07267v1 (2019).

21. G. A. Margulis, Arithmeticity of the irreducible lattices in the semisimple groups of rank greater than 1, Invent. Math. 76 (1984), no. 1, 93-120. MR 739627

22. _ Discrete subgroups of semisimple Lie groups, Ergebnisse der Mathematik und ihrer Grenzgebiete (3) [Results in Mathematics and Related Areas (3)], vol. 17, Springer-Verlag, Berlin, 1991. MR 1090825 (92h:22021)

23. D. B. McReynolds and A. W. Reid, The genus spectrum of hyperbolic 3-manifolds, Math. Research Letters 21 (2014), 169-185.

24. Shahar Mozes and Nimish Shah, On the space of ergodic invariant measures of unipotent flows, Ergodic Theory Dynam. Systems 15 (1995), no. 1, 149-159. MR 1314973

25. Alan Reid, Totally geodesic surfaces in hyperbolic 3-manifolds, Proceedings of the Edinburgh Mathematical Society 34 (1991), 77 - 88.

26. Atle Selberg, On discontinuous groups in higher-dimensional symmetric spaces, Contributions to function theory (internat. Colloq. Function Theory, Bombay, 1960), Tata Institute of Fundamental Research, Bombay, 1960, pp. 147-164. MR 0130324

27. Andre Weil, On discrete subgroups of lie groups, Annals of Mathematics 72 (1960), no. 2, 369-384.

28. _ Remarks on the cohomology of groups, Annals of Mathematics 80 (1964), no. $1,149-157$.

G.M.: Yale University, Mathematics Dept., PO Box 208283, New Haven, CT 06520, USA

E-mail address: gregorii.margulis@yale.edu

A.M.: Department of Mathematics, The University of California, San Diego, CA 92093, USA

E-mail address: ammohammadi@ucsd.edu 